Cochrane Database of Systematic Reviews

\title{
Drug-eluting balloon angioplasty versus uncoated balloon angioplasty for the treatment of in-stent restenosis of the femoropopliteal arteries (Review)
}

Kayssi A, Al-Jundi W, Papia G, Kucey DS, Forbes T, Rajan DK, Neville R, Dueck AD

Kayssi A, Al-Jundi W, Papia G, Kucey DS, Forbes T, Rajan DK, Neville R, Dueck AD.

Drug-eluting balloon angioplasty versus uncoated balloon angioplasty for the treatment of in-stent restenosis of the femoropopliteal arteries.

Cochrane Database of Systematic Reviews 2019, Issue 1. Art. No.: CD012510.

DOI: 10.1002/14651858.CD012510.pub2. 
TABLE OF CONTENTS

HEADER 1

ABSTRACT

PLAIN LANGUAGE SUMMARY ....

SUMMARY OF FINDINGS

BACKGROUND

OBJECTIVES

METHODS

RESULTS

Figure 1.

Figure 2.

Figure 3.

DISCUSSION

AUTHORS' CONCLUSIONS

ACKNOWLEDGEMENTS

REFERENCES

CHARACTERISTICS OF STUDIES

DATA AND ANALYSES

Analysis 1.1. Comparison 1 Drug-eluting balloon (DEB) versus uncoated balloon angioplasty at 1 month, Outcome 1 Improvement $\geq 1$ Rutherford category.

Analysis 2.1. Comparison 2 Drug-eluting balloon (DEB) versus uncoated balloon angioplasty at 6 months, Outcome 1 Target lesion revascularization.

Analysis 2.2. Comparison 2 Drug-eluting balloon (DEB) versus uncoated balloon angioplasty at 6 months, Outcome 2 Binary restenosis.

Analysis 2.3. Comparison 2 Drug-eluting balloon (DEB) versus uncoated balloon angioplasty at 6 months, Outcome 3 Death. ..

Analysis 2.4. Comparison 2 Drug-eluting balloon (DEB) versus uncoated balloon angioplasty at 6 months, Outcome 4 Improvement $\geq 1$ Rutherford category.

Analysis 3.1. Comparison 3 Drug-eluting balloon (DEB) versus uncoated balloon angioplasty at 12 months, Outcome 1 Binary restenosis.

Analysis 3.2. Comparison 3 Drug-eluting balloon (DEB) versus uncoated balloon angioplasty at 12 months, Outcome 2 Death. .

Analysis 3.3. Comparison 3 Drug-eluting balloon (DEB) versus uncoated balloon angioplasty at 12 months, Outcome 3 Improvement $\geq 1$ Rutherford category.

Analysis 4.1. Comparison 4 Drug-eluting balloon (DEB) versus uncoated balloon angioplasty at 24 months, Outcome 1 Target lesion revascularization.

Analysis 4.2. Comparison 4 Drug-eluting balloon (DEB) versus uncoated balloon angioplasty at 24 months, Outcome 2 Death. . APPENDICES

CONTRIBUTIONS OF AUTHORS

DECLARATIONS OF INTEREST

SOURCES OF SUPPORT

DIFFERENCES BETWEEN PROTOCOL AND REVIEW

NOTES

INDEX TERMS 
[Intervention Review]

\title{
Drug-eluting balloon angioplasty versus uncoated balloon angioplasty for the treatment of in-stent restenosis of the femoropopliteal arteries
}

\author{
Ahmed Kayssi ${ }^{1}$, Wissam Al-Jundi ${ }^{1}$, Giuseppe Papia ${ }^{1}$, Daryl S Kucey ${ }^{1}$, Thomas Forbes ${ }^{2}$, Dheeraj K Rajan ${ }^{3}$, Richard Neville ${ }^{4}$, Andrew D \\ Dueck ${ }^{1}$ \\ 1Division of Vascular Surgery, Sunnybrook Health Sciences Centre, University of Toronto, Toronto, Canada. 2 Division of Vascular Surgery, \\ Toronto General Hospital, University of Toronto, Toronto, Canada. ${ }^{3}$ Division of Vascular and Interventional Radiology, University of \\ Toronto, Toronto, Canada. ${ }^{4}$ Inova Heart and Vascular Institute, Falls Church, Virginia, USA
}

Contact address: Ahmed Kayssi, Division of Vascular Surgery, Sunnybrook Health Sciences Centre, University of Toronto, Room H287, 2075 Bayview Avenue, Toronto, ON, M4N 3M5, Canada. Ahmed.Kayssi@sunnybrook.ca.

Editorial group: Cochrane Vascular Group.

Publication status and date: New, published in Issue 1, 2019.

Citation: Kayssi A, Al-Jundi W, Papia G, Kucey DS, Forbes T, Rajan DK, Neville R, Dueck AD. Drug-eluting balloon angioplasty versus uncoated balloon angioplasty for the treatment of in-stent restenosis of the femoropopliteal arteries. Cochrane Database of Systematic Reviews 2019, Issue 1. Art. No.: CD012510. DOI: 10.1002/14651858.CD012510.pub2.

Copyright @ 2019 The Cochrane Collaboration. Published by John Wiley \& Sons, Ltd.

\section{A B S T R A C T}

\section{Background}

Stents are placed in the femoropopliteal arteries for numerous reasons, such as atherosclerotic disease, the need for dissection, and perforation of the arteries, and can become stenosed with the passage of time. When a stent develops a flow-limiting stenosis, this process is known as "in-stent stenosis." It is thought that in-stent restenosis is caused by a process known as "intimal hyperplasia" rather than by the progression of atherosclerotic disease. Management of in-stent restenosis may include performing balloon angioplasty, deploying another stent within the stenosed stent to force it open, and creating a bypass to deliver blood around the stent. The role of drug-eluting technologies, such as drug-eluting balloons (DEBs), in the management of in-stent restenosis is unclear. Drug-eluting balloons might function by coating the inside of stenosed stents with cytotoxic chemicals such as paclitaxel and by inhibiting the hyperplastic processes responsible for in-stent restenosis. It is important to perform this systematic review to evaluate the efficacy of DEB because of the potential for increased expenses associated with DEBs over uncoated balloon angioplasty, also known as plain old balloon angioplasty (POBA).

\section{Objectives}

To assess the safety and efficacy of DEBs compared with uncoated balloon angioplasty in people with in-stent restenosis of the femoropopliteal arteries as assessed by criteria such as amputation-free survival, vessel patency, target lesion revascularization, binary restenosis rate, and death. We define "in-stent restenosis" as 50\% or greater narrowing of a previously stented vessel by duplex ultrasound or angiography.

\section{Search methods}

The Cochrane Vascular Information Specialist searched the Cochrane Vascular Specialised Register, CENTRAL, MEDLINE, Embase, and CINAHL databases and the World Health Organization International Clinical Trials Registry Platform and ClinicalTrials.gov trials registers to November 28, 2017. Review authors also undertook reference checking to identify additional studies.

\section{Selection criteria}

We included all randomized controlled trials that compared DEBs versus uncoated balloon angioplasty for treatment of in-stent restenosis in the femoropopliteal arteries. 


\section{Data collection and analysis}

Two review authors (AK, WA) independently selected appropriate trials and performed data extraction, assessment of trial quality, and data analysis. The senior review author (AD) adjudicated any disagreements.

\section{Main results}

Three trials that randomized a combined total of 263 participants met the review inclusion criteria. All three trials examined the treatment of symptomatic in-stent restenosis within the femoropopliteal arteries. These trials were carried out in Germany and Austria and used paclitaxel as the agent in the drug-eluting balloons. Two of the three trials were industry sponsored. Two companies manufactured the drug-eluting balloons (Eurocor, Bonn, Germany; Medtronic, Fridley, Minnesota, USA). The trials examined both anatomical and clinical endpoints. We noted heterogeneity in the frequency of bailout stenting deployment between studies as well as in the dosage of paclitaxel applied by the DEBs. Using GRADE assessment criteria, we determined that the certainty of evidence presented was very low for the outcomes of amputation, target lesion revascularization, binary restenosis, death, and improvement of one or more Rutherford categories. Most participants were followed up to 12 months, but one trial followed participants for up to 24 months.

Trial results show no difference in the incidence of amputation between DEBs and uncoated balloon angioplasty. DEBs showed better outcomes for up to 24 months for target lesion revascularization (odds ratio (OR) $0.05,95 \%$ confidence Interval (CI) 0.00 to 0.92 at six months; OR $0.24,95 \% \mathrm{Cl} 0.08$ to 0.70 at 24 months) and at six and 12 months for binary restenosis (OR $0.28,95 \% \mathrm{Cl} 0.14$ to 0.56 at six months; OR $0.34,95 \% \mathrm{Cl} 0.15$ to 0.76 at 12 months). Participants treated with DEBs also showed improvement of one or more Rutherford categories at six and 12 months (OR $1.81,95 \% \mathrm{Cl} 1.02$ to 3.21 at six months; OR $2.08,95 \% \mathrm{Cl} 1.13$ to 3.83 at 12 months). Data show no clear differences in death between DEBs and uncoated balloon angioplasty. Data were insufficient for subgroup or sensitivity analyses to be conducted.

\section{Authors' conclusions}

Based on a meta-analysis of three trials with 263 participants, evidence suggests an advantage for DEBs compared with uncoated balloon angioplasty for anatomical endpoints such as target lesion revascularization (TLR) and binary restenosis, and for one clinical endpoint - improvement in Rutherford category post intervention for up to 24 months. However, the certainty of evidence for all these outcomes is very low due to the small number of included studies and participants and the high risk of bias in study design. Adequately powered and carefully constructed randomized controlled trials are needed to adequately investigate the role of drug-eluting technologies in the management of in-stent restenosis.

\section{PLAIN LANGUAGE SUMMARY}

\section{Drug-eluting balloon angioplasty versus uncoated balloon angioplasty for stenosis of stents in lower limb arteries}

\section{Background}

Many people have disease in their leg arteries that may develop into a blockage and lead to loss of circulation and subsequent pain, skin ulcers, and amputation of the leg. In an effort to prevent lack of blood from harming the leg, procedures can be performed to bypass the blocked artery using a vein or an artificial graft, or to cross it with a wire and open it with a balloon, then place a stent to help prevent the blockage from happening again. Although stents are very strong and can last a long time, it is possible that a stent inside an artery might at times become narrower, and eventually can become blocked. This process is known as "in-stent restenosis." A blocked stent can be treated in several ways, such as sucking out the clot or ballooning it, or placing another stent inside the stent that failed. Although all treatment options offer advantages and disadvantages, there have been advancements in the technologies available to treat this problem. One of these advancements requires covering the balloon used to treat the stent blockage with a cytotoxic drug used in chemotherapy, to slow down the blockage process after the stent is treated. Such specially prepared balloons, known as "drug-eluting balloons," have shown encouraging results for treating patients with artery disease in the leg.

\section{Review question}

The goal of this review was to determine how drug-eluting balloon (DEB) angioplasty compares with traditional uncoated balloon angioplasty, also known as plain old balloon angioplasty (POBA), for the treatment of in-stent restenosis in stents placed in leg arteries.

\section{Study characteristics}

Our review included three clinical trials that randomized 263 participants (most recent search - November 28, 2017). Trials included leg arteries at and above the knee and were carried out in Europe; all used DEBs that contained the chemical known as "paclitaxel." Two companies manufactured the DEBs: Eurocor and Medtronic. Most study participants were followed for six or more months; this is called "follow-up."

\section{Key results}

Results showed that DEBs were not better for participants than uncoated balloon angioplasty with regard to the need for amputation. At 24 months of follow-up, DEBs were associated with fewer target lesion revascularizations, which refers to the need to perform a procedure on a stent that had already been treated with a DEB or an uncoated balloon angioplasty for in-stent restenosis. DEBs were also found to have better binary restenosis rates, which refers to the percentage of treated stents that develop new stenosis after they have been treated with a DEB or an uncoated balloon angioplasty. Finally, more people who were treated with DEBs described improvement in their leg 
symptoms, as measured by a change in their Rutherford category. DEBs were not found to be better for participants than uncoated balloon angioplasty with regard to patient death.

\section{Certainty of the evidence}

The certainty of the evidence presented was very low because we identified only three studies with small numbers of participants, and because many participants in those studies were lost to follow-up. Furthermore, risk of performance and attrition bias was significant, as was risk of other biases, due to lack of accounting for the type of stent treated and the need for bailout stenting. 


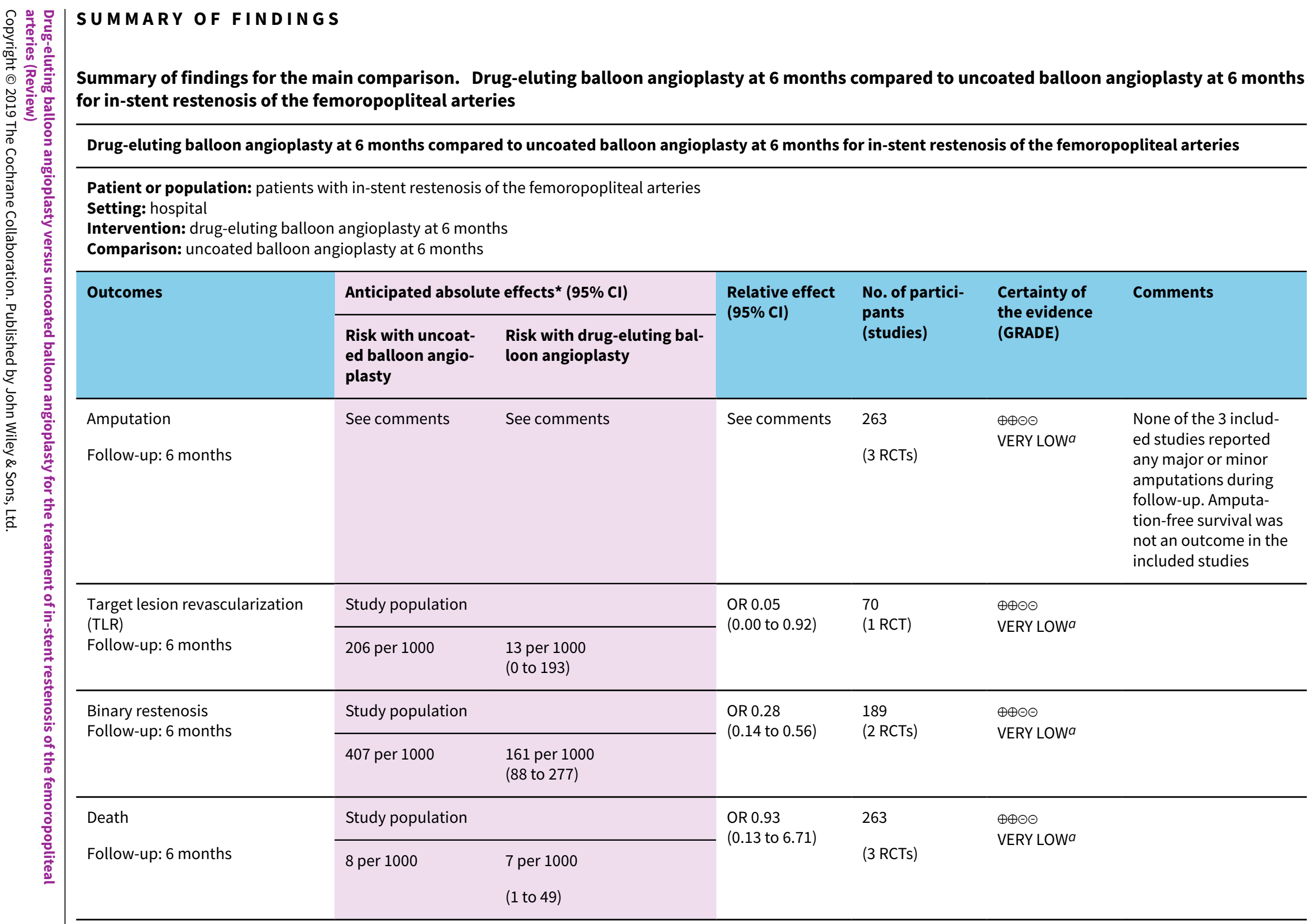




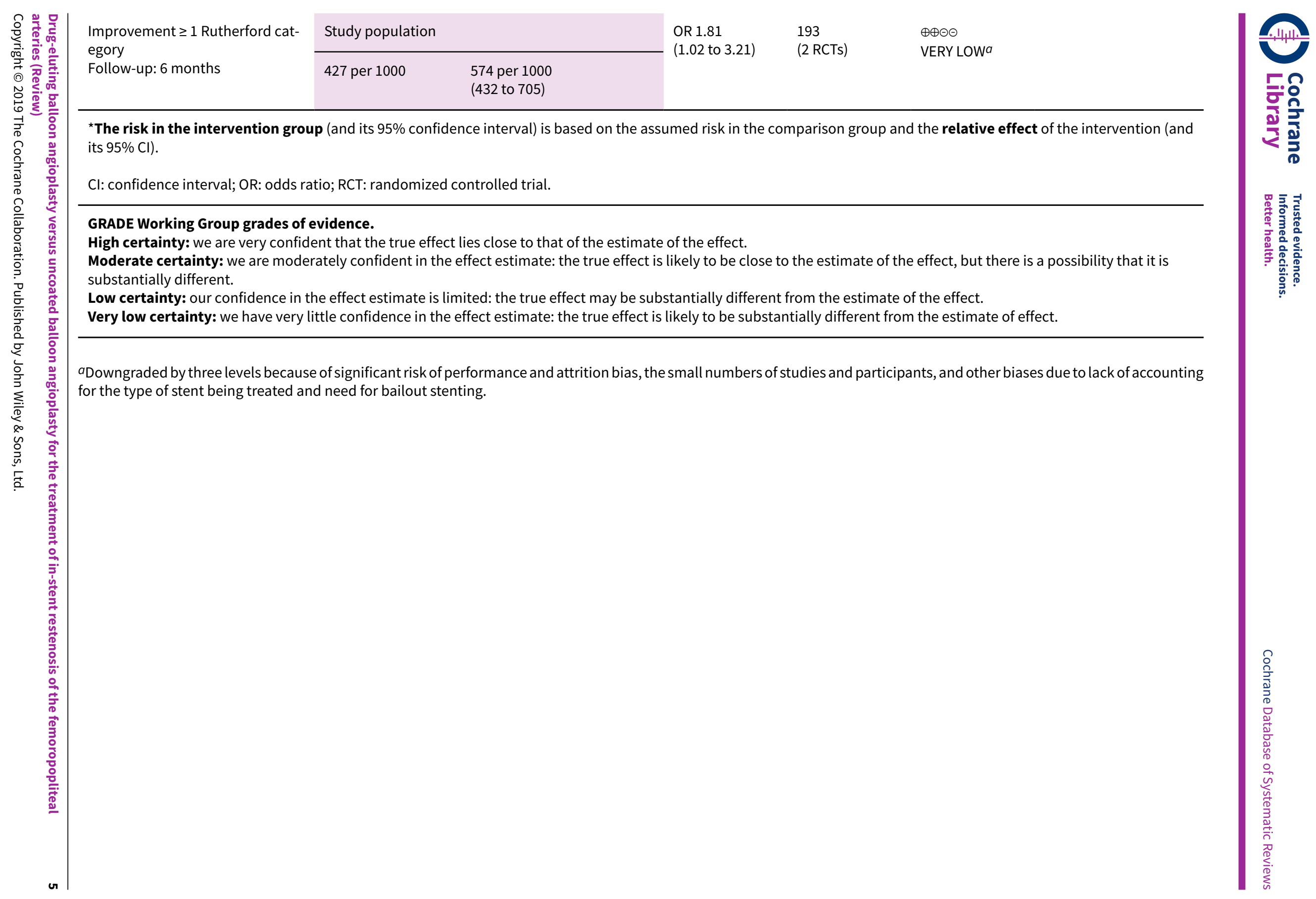




\section{B A C K G R O U N D}

\section{Description of the condition}

Peripheral arterial disease (PAD) of the lower extremities is closely associated with atherosclerosis, a progressive and chronic disease process that leads to hardened vessels and decreased blood flow (Stary 1994). Although many people with PAD of the lower extremities are asymptomatic, others present with a variety of symptoms, including pain with walking (intermittent claudication), pain at rest, and tissue loss in the affected limbs (McDermott 2001). PAD is a highly prevalent condition that increases with age and occurs in more than $10 \%$ of individuals above the age of 60 (Criqui 2015).

Numerous treatment modalities have been used for the management of PAD of the lower extremities. Medical therapies aim to arrest the progression of atherosclerotic disease and improve clinical outcomes by modifying patient risk factors (Ali 2012). Open surgical and endovascular interventions are used in conjunction with medical therapies to improve blood delivery to ischemic tissues by bypassing or otherwise opening arterial blockages with wires, catheters, and other specialized devices (Conte 2015).

Of the growing number of interventions available for treating patients with lower extremity PAD, deployment of stents across a stenotic or occluded arterial segment has become a routine practice in certain circumstances, such as with long lesions and those that do not respond well to balloon angioplasty alone (OHTAC 2010). Stenting provides the mechanical support required to maintain the patency of arterial lesions, but its effectiveness is limited by the occurrence of neointimal proliferation, known as "intimal hyperplasia," and by overgrowth of the inner lining of the artery within the stent. This process, known as "in-stent restenosis," may limit blood flow through the stent and may sometimes result in recurrence of ischemic symptoms (Alfonso 2014).

\section{Description of the intervention}

Numerous endovascular interventions have been described for the treatment of in-stent restenosis, including repeat uncoated balloon angioplasty, also known as "plain old balloon angioplasty (POBA)" alone, cutting balloon angioplasty, angioplasty with drug-eluting balloons (DEBs), repeat stenting, and atherectomy (Singh 2014).

For all of these interventions, a wire is used first to cross the stenotic or occlusive lesion within the stent before treatment can be administered. Use of DEBs requires pre-dilation of the stenotic lesion with a plain balloon. A second balloon is then used to coat the inside of the stent with a cytotoxic chemical in the hope of reducing neointimal proliferation.

\section{How the intervention might work}

Uncoated balloon angioplasty has been used widely and successfully for the treatment of lower extremity PAD and in-stent restenosis, but its use is limited by recurrence of the neointimal hyperplastic process. DEBs address this limitation by applying a chemical to the stent that exerts an antiproliferative effect and arrests the restenotic process (Waksman 2009).

The most commonly used DEB cytotoxic agent is the taxane paclitaxel, a chemotherapeutic medication that exerts its cytotoxic effect by stabilizing microtubules (Marupudi 2007). Paclitaxel is a safe and effective DEB agent for treatment of PAD and has been used successfully to decrease the incidence of binary restenosis in femoropopliteal vessels (Canaud 2014).

\section{Why it is important to do this review}

Numerous prospective studies have compared DEB versus uncoated balloon angioplasty for treatment of in-stent restenosis. DEBs have shown promising results in the treatment of coronary in-stent restenosis (Indermuehle 2013). However, the certainty of evidence for their use in femoropopliteal vessels remains unclear. Furthermore, DEB technologies are more expensive and are less widely available than uncoated balloon angioplasty. Our review seeks to assess the efficacy of this intervention and to determine whether it is associated with improved clinical outcomes.

\section{O B JECTIVES}

To assess the safety and efficacy of DEBs compared with uncoated balloon angioplasty in people with in-stent restenosis of the femoropopliteal arteries as assessed by criteria such as amputation-free survival, vessel patency, target lesion revascularization, binary restenosis rate, and death. We define "in-stent restenosis" as 50\% or greater narrowing of a previously stented vessel by duplex ultrasound or angiography.

\section{METHODS}

\section{Criteria for considering studies for this review \\ Types of studies}

We included randomized controlled trials (RCTs) that compared drug-eluting balloons (DEBs) versus uncoated balloon angioplasty for in-stent restenosis of the femoropopliteal arteries. We define "in-stent restenosis" as 50\% or greater narrowing of a previously stented vessel by duplex ultrasound or angiography.

\section{Types of participants}

We included people undergoing drug-eluting or uncoated balloon angioplasty for treatment of in-stent restenosis of the femoropopliteal arteries.

\section{Types of interventions}

We sought to compare DEBs versus uncoated balloon angioplasty for treatment of in-stent restenosis of femoropopliteal arteries. Analysis excluded hybrid studies in which DEBs are utilized simultaneously with open surgery, as well as studies that include atherectomy procedures, cutting balloon angioplasty, vascular brachytherapy, and repeat stenting with plain or drug-eluting stents for management of in-stent restenosis.

\section{Types of outcome measures}

\section{Primary outcomes}

- Amputation-free survival, defined as the probability of being alive without loss of the affected lower extremity

- Vessel patency, both primary and secondary, measured by late lumen loss, target lesion revascularization (TLR), and rate of binary restenosis, defined as $50 \%$ or greater narrowing of the vessel by duplex ultrasound or angiography

- Death 


\section{Secondary outcomes}

- Change in Rutherford category of PAD

- Change in the Society for Vascular Surgery Wound, Ischemia, and Foot Infection (WIfl) stage (Mills 2014)

- Change in ankle-brachial index (ABI)

- Change in quality of life (QoL) scores, measured by validated instruments such as Short Form 36 (SF-36)

- Change in functional walking ability, measured by change in walking distance (on a treadmill or by other walking test) or use of a walking impairment questionnaire

\section{Search methods for identification of studies}

We applied no language, publication year, or publication status restrictions.

\section{Electronic searches}

The Cochrane Vascular Information Specialist searched the following databases for relevant trials.

- Cochrane Vascular Specialised Register (February 2, 2017).

- Cochrane Central Register of Controlled Trials (CENTRAL; 2017, Issue 1), in the Cochrane Library, via the Cochrane Register of Studies Online.

See Appendix 1 for details of the search strategy that were used to search CENTRAL.

The Information Specialist also searched the following trials registries for details of ongoing and unpublished studies (February 2, 2017).

- ClinicalTrials.gov (clinicaltrials.gov).

- World Health Organization International Clinical Trials Registry Platform (who.int/trialsearch).

- International Standard Randomized Controlled Trials Number (ISRCTN) Register (isrctn.com/).

The Information Specialist subsequently conducted top-up searches of the following databases.

- Cochrane Vascular Specialised Register via the Cochrane Register of Studies (CRS-Web searched from January 1, 2017, to November 28, 2017).

- Cochrane Central Register of Controlled Trials (CENTRAL), in the Cochrane Library, via the Cochrane Register of Studies Online (CRSO; 2017, Issue 10).

- MEDLINE (Ovid MEDLINE ${ }^{\circledR}$ Epub Ahead of Print, In-Process \& Other Non-Indexed Citations, Ovid MEDLINE ${ }^{\circledR}$ Daily, and Ovid MEDLINE ${ }^{\circledR}$ ) (searched from January 1, 2017, to November 28, 2017).

- Embase Ovid (searched from January 1, 2017, to November 28, 2017).

- Cumulative Index to Nursing and Allied Health Literature (CINAHL) Ebsco (searched from January 1, 2017, to November 28, 2017).

- Allied and Complementary Medicine Database (AMED) Ovid (searched from January 1, 2017, to November 28, 2017).

The Information Specialist modeled search strategies for the listed databases on the search strategy designed for CENTRAL. When appropriate, the search strategies were combined with adaptations of the highly sensitive search strategy designed by Cochrane for identifying RCTs and controlled clinical trials (as described in Chapter 6, of the Cochrane Handbook for Systematic Reviews of Interventions; Higgins 2011). We have provided search strategies for the major databases in Appendix 3.

The Information Specialist also performed top-up searches of the following trials registries on November 28, 2017.

- World Health Organization International Clinical Trials Registry Platform (who.int/trialsearch).

- ClinicalTrials.gov (clinicaltrials.gov).

\section{Searching other resources}

We further examined the bibliographies of studies identified in our search to identify other relevant articles.

\section{Data collection and analysis}

\section{Selection of studies}

Two review authors (AK, WA) independently selected trials for inclusion in this review from studies identified by the search. A third review author (AD) assessed these trials, determined their suitability, and adjudicated disagreements between the first two review authors. We have outlined in the Criteria for considering studies for this review section the inclusion criteria used to determine suitability.

\section{Data extraction and management}

Two review authors (AK, WA) extracted relevant data from the included studies. We collected participant demographics (age, gender, PAD comorbidities, Rutherford PAD category, and baseline $A B I$ ), interventions (types and duration of DEBs and uncoated balloon angioplasty), and outcomes (as specified in the Criteria for considering studies for this review section). A third review author (AD) cross-checked data.

Statistical analysis complied with the standard methods of Cochrane Vascular. We used the computer software package Review Manager 5.3 (RevMan) to perform all statistical analyses and to generate figures (Review Manager 2014).

\section{Assessment of risk of bias in included studies}

Two review authors (AK, WA) carried out a thorough risk of bias assessment of all included studies using the Cochrane tool for assessing risk of bias (Higgins 2011). This tool assesses bias in six different domains, with each domain receiving a score of high, low, or unclear, depending on each review author's judgement. A third review author (AD) adjudicated disagreements. We contacted study authors when we needed clarification to better assess risk of bias.

\section{Measures of treatment effect}

We used the mean difference (MD) to calculate and report continuous outcome measures, such as change in QoL scores. If included studies used different scales, we calculated the standardized mean difference (SMD) instead. We also calculated the associated $95 \%$ confidence interval $(\mathrm{Cl})$ between the two treatment groups. We calculated and reported dichotomous (binary) outcome measures, such as target lesion revascularization, using the hazard ratio $(\mathrm{HR})$ or the risk ratio (RR) with associated $95 \% \mathrm{Cl}$, depending

Drug-eluting balloon angioplasty versus uncoated balloon angioplasty for the treatment of in-stent restenosis of the femoropopliteal 
on reported data. We carried out analyses at different time points, as reported by the trials. We based our calculations on an intentionto-treat approach.

\section{Unit of analysis issues}

The unit of analysis was the treated limb for anatomical outcomes such as late lumen loss or binary restenosis. Conversely, the unit of analysis was the patient for outcomes such as quality of life or death.

\section{Dealing with missing data}

We contacted study authors to inquire about missing or incomplete data. In the event that a significant quantity of data was missing from an included study and attempts to contact study authors were unsuccessful, we assessed the risk of bias associated with including the study in the meta-analysis and performed a sensitivity analysis.

\section{Assessment of heterogeneity}

We assessed interstudy heterogeneity by using a forest plot (Schünemann 2011). We calculated $\mathrm{Chi}^{2}$ and $\mathrm{I}^{2}$ values to measure the amount of heterogeneity (Higgins 2003). $1^{2}$ values less than $50 \%$ indicated low heterogeneity, $\mathrm{I}^{2}$ values between $50 \%$ and $75 \%$ indicated moderate heterogeneity, and $\mathrm{I}^{2}$ values greater than $75 \%$ indicated significant heterogeneity (Deeks 2011).

\section{Assessment of reporting biases}

We planned to construct a funnel plot to assess publication bias when 10 or more studies were available for a particular outcome (Sterne 2011).

\section{Data synthesis}

To calculate the pooled treatment effect data, we used randomeffects or fixed-effect models, depending on the degree of interstudy heterogeneity. If the calculated degree of interstudy heterogeneity was significant (defined as $I^{2}$ greater than $75 \%$ ), we used the random-effects model. Otherwise, we used the fixed-effect model. We calculated $95 \% \mathrm{Cls}$ for continuous and dichotomous outcome variables, as detailed above. We created a forest plot for each treatment effect as per Cochrane Vascular guidelines.

\section{Subgroup analysis and investigation of heterogeneity}

We planned to perform subgroup analyses by type of DEB, type and dose of pharmacological agent used in the DEB, and clinical severity of PAD, as defined by the change in Rutherford category or the WIfl stage. We also planned to perform subgroup analyses for people with diabetes and for the types of antiplatelet agents prescribed for participants in the included trials.

\section{Sensitivity analysis}

We planned to exclude studies at high risk of bias from the pooled analysis and to repeat the analysis to assess their impact. We planned to performed a sensitivity analysis by sequentially excluding studies at high risk of bias.

\section{"Summary of findings"}

We prepared a "Summary of findings" table using the GRADEpro Guideline Development Tool to present the main findings of the review for the time point at which the most relevant data were available from the included studies (www.gradepro.org; Atkins 2004). The population consisted of people with PAD, and we compared DEBs versus uncoated balloon angioplasty for treatment of in-stent restenosis of femoropopliteal arteries. We included in our "Summary of findings" table the main outcomes listed under Types of outcome measures that we considered essential for decision-making. We evaluated the certainty of the evidence using the GRADE approach (Schünemann 2011b). We assigned one of four levels of quality: high, moderate, low, or very low, based on overall risk of bias, directness of evidence, inconsistency of results, precision of estimates, and risk of publication bias, as previously described (Higgins 2011).

\section{RES U L T S}

\section{Description of studies}

\section{Results of the search}

Please see Figure 1 for search results. 
Figure 1. Study flow diagram.

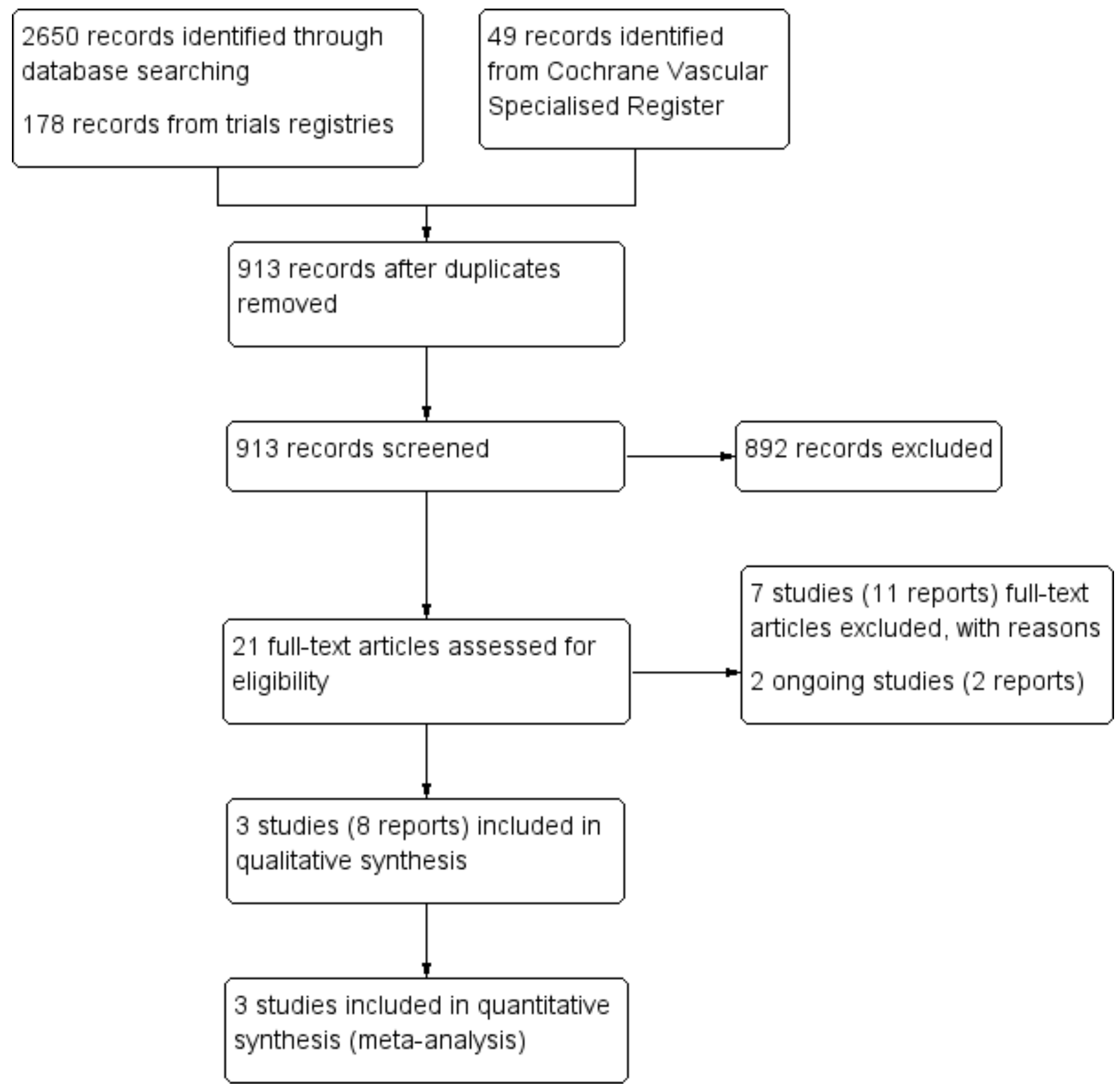

\section{Included studies}

This review includes three randomized controlled trials that compared DEB versus uncoated balloon angioplasty for treatment of symptomatic in-stent restenosis of the femoropopliteal arteries (FAIR; ISAR-PEBIS; PACUBA). The Characteristics of included studies tables present the details of those studies.

The three included studies were conducted in Germany - FAIR; ISAR-PEBIS - and in Austria - PACUBA. All studies included patients with in-stent restenosis in the femoropopliteal arteries and used paclitaxel-coated balloons, although at different dosages (Medtronic IN.PACT Admiral $3.5 \mathrm{mcg} / \mathrm{mm}^{2}$ in FAIR and ISAR-PEBIS vs Eurocor Freeway $3 \mathrm{mcg} / \mathrm{mm}^{2}$ in PACUBA). We also identified two ongoing trials (Copa Cabana; TRC-14004848). Details of the ongoing trials are presented in the Characteristics of ongoing studies tables.
We contacted the authors of all included and ongoing studies to request study data.

\section{Excluded studies}

We excluded seven studies from our review (Bosiers 2015; ConSeQuent; DEBATE-ISR; EXCITE ISR; NCT00481780; NCT02832024; RELINE), and we listed reasons for exclusion in the Characteristics of excluded studies tables. We excluded studies because they compared stenting in Bosiers 2015 and RELINE, laser atherectomy in EXCITE ISR, or cutting balloon angioplasty in NCT00481780 versus uncoated balloon angioplasty for in-stent restenosis. The ConSeQuent trial compared DEB versus uncoated balloon angioplasty for native vessel restenosis rather than in-stent restenosis. The DEBATE-ISR study compared a prospective cohort of patients receiving DEB therapy for in-stent restenosis against

Drug-eluting balloon angioplasty versus uncoated balloon angioplasty for the treatment of in-stent restenosis of the femoropopliteal 
a historical cohort of diabetic patients. Finally, the NCT02832024 study compared stent deployment versus atherectomy versus uncoated balloon angioplasty alone for in-stent restenosis.

\section{Risk of bias in included studies}

Please refer to the "Risk of bias" tables in the Characteristics of included studies tables and summary results in Figure 2 and Figure

\section{Figure 2. Risk of bias graph: review authors' judgements about each risk of bias item presented as percentages} across all included studies.

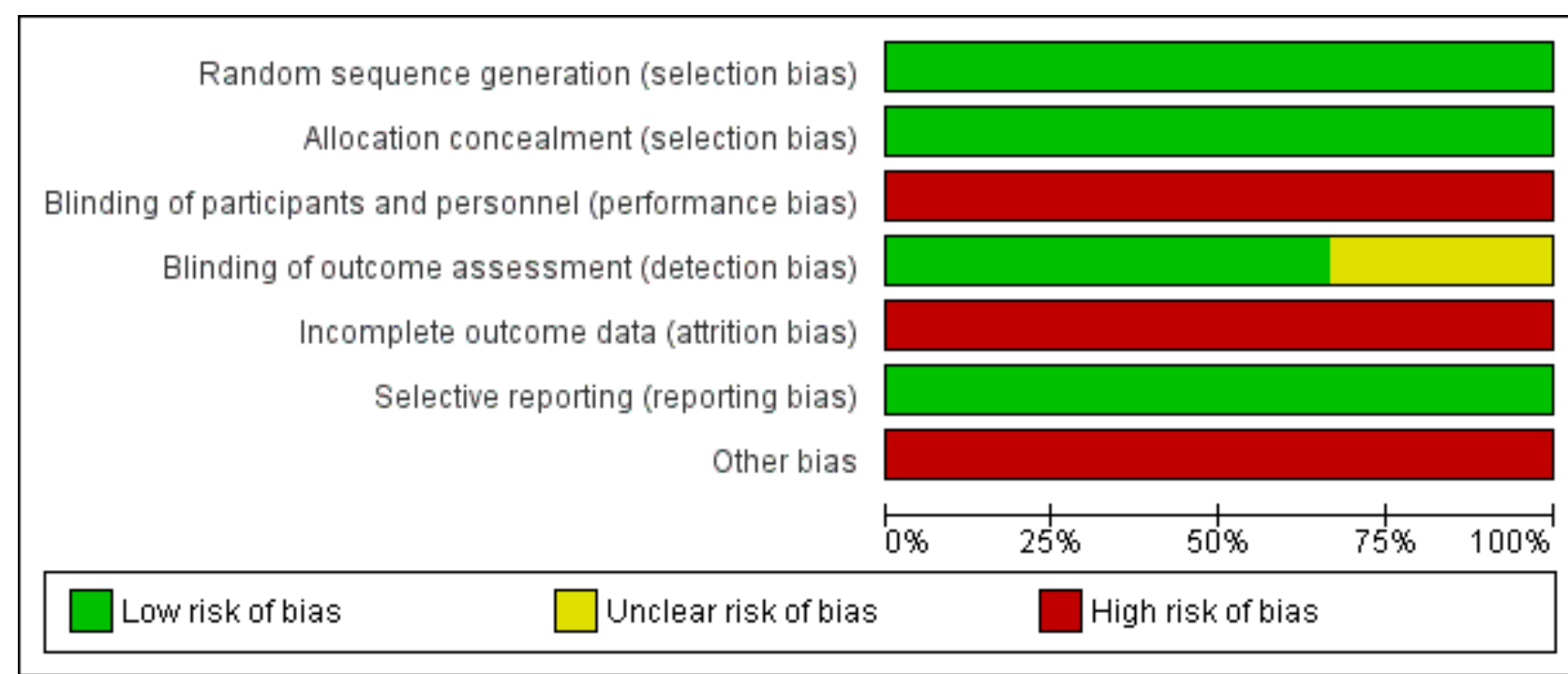

Drug-eluting balloon angioplasty versus uncoated balloon angioplasty for the treatment of in-stent restenosis of the femoropopliteal 
Figure 3. Risk of bias summary: review authors' judgements about each risk of bias item for each included study.

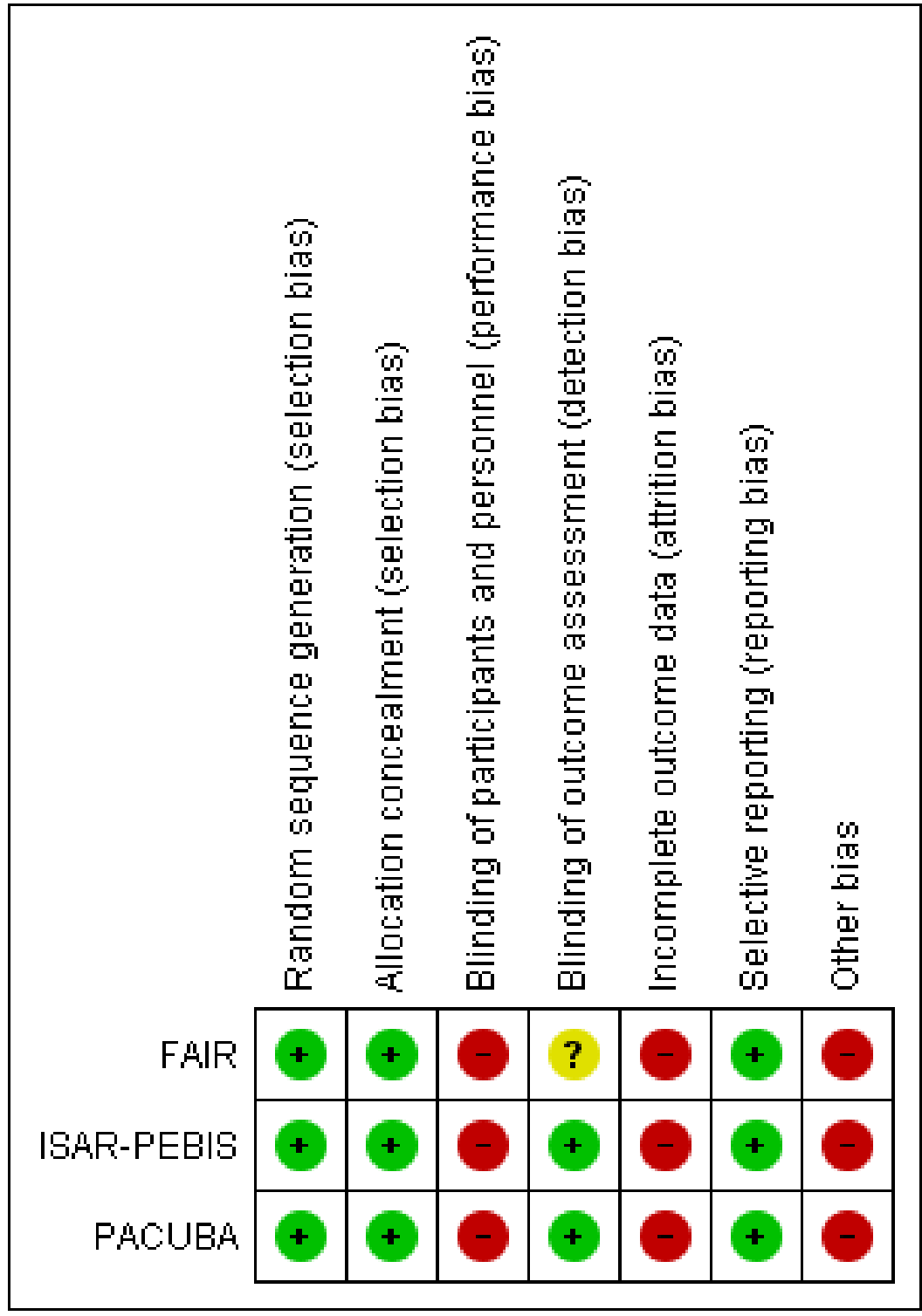

\section{Allocation}

The included studies were at low risk of selection bias in random sequence generation or allocation concealment. The FAIR trial used a block randomization study design with a block size of 10 and an allocation ratio of $1: 1$, and concealed the allocation sequence from investigators by using sequentially numbered and opaque sealed envelopes. The ISAR-PEBIS study used computer sequence generation to randomize participants after making the decision to proceed with an intervention. These researchers similarly accomplished the allocation of treatment by using sealed and opaque envelopes. The PACUBA trial investigators used a 1:1 randomization protocol with computer-generated sequences and concealed allocation via use of sealed envelopes.

\section{Blinding}

All three included studies were at high risk of performance bias because the operators were not blinded (FAIR; ISAR-PEBIS; PACUBA). Conversely, however, the outcomes assessors in two trials were blinded to the treatment arm (ISAR-PEBIS; PACUBA), placing them at low risk for detection bias. It is unclear whether the outcomes assessors in the FAIR trial were blinded to the treatment arm.

\section{Incomplete outcome data}

All three included studies were at high risk of attrition bias due to loss to follow-up (FAIR; ISAR-PEBIS; PACUBA). In the FAIR trial, approximately one-third of participants were lost to follow-up at 12 months. Similarly, in the ISAR-PEBIS trial, approximately $25 \%$ of DEB participants and $21 \%$ of uncoated balloon angioplasty participants did not undergo angiography as per the trial's primary endpoint requirement. In the PACUBA trial, eight participants in the DEB group $(23 \%)$ and six in the uncoated balloon angioplasty group (15\%) were lost to follow-up.

Drug-eluting balloon angioplasty versus uncoated balloon angioplasty for the treatment of in-stent restenosis of the femoropopliteal 


\section{Selective reporting}

All three included studies were at low risk for reporting bias (FAIR; ISAR-PEBIS; PACUBA). Trial authors reported all outcomes that were specified in the study protocols.

\section{Other potential sources of bias}

Two major sources of bias in all included trials were specifying the types of stents treated for in-stent restenosis and addressing the differences in bailout stenting requirements between treatment arms (FAIR; ISAR-PEBIS; PACUBA).

Study authors did not specify whether stents that were treated were bare-metal, covered, or drug-eluting, nor did they stratify the results by length of the stent or duration of time since stent implantation.

The other major source of bias is the disparity in the incidence of bailout stenting between treatment arms in all three included studies. In the FAIR study, four uncoated balloon angioplasty participants received bailout stenting compared with one participant in the DEB arm. Conversely, in the ISAR-PEBIS study, $26 \%$ of DEB participants received bailout stenting compared with $6 \%$ of uncoated balloon angioplasty participants $(P=0.02)$. In the PACUBA study, four DEB participants received bailout stenting compared with one uncoated balloon angioplasty participant. Authors of the included studies did not stratify reported results by bailout stent implantation status.

Furthermore, study populations in the PACUBA trial were not similar, as the uncoated balloon angioplasty group reported more advanced lesions (Tosaka II and TASC B and C) than were noted in the DEB group (67\% Tosaka class II lesions in the uncoated balloon angioplasty group vs $46 \%$ in the DEB group, $36 \%$ TASC B and $26 \%$ TASC C lesions in the uncoated balloon angioplasty group vs $23 \%$ and $14 \%$ in the uncoated balloon angioplasty group, respectively).

\section{Effects of interventions}

See: Summary of findings for the main comparison Drugeluting balloon angioplasty at 6 months compared to uncoated balloon angioplasty at 6 months for in-stent restenosis of the femoropopliteal arteries

\section{Primary outcomes}

\section{Amputation-free survival}

None of the included studies reported amputation-free survival (FAIR; ISAR-PEBIS; PACUBA).

\section{Amputation}

The three included studies reported no major or minor lower extremity amputations at one, six, 12, or 24 months of follow-up (FAIR; ISAR-PEBIS; PACUBA). We judged the certainty of evidence to be very low, however, as none of the included trials were powered to detect differences in clinical endpoints such as amputation, and all of the included trials were at significant risk of performance and attrition bias.

\section{Vessel patency}

\section{Primary vessel patency}

One study reported primary vessel patency (PACUBA). Study authors defined primary vessel patency as a $<50 \%$ reduction in diameter stenosis by colored duplex ultrasound and reported no statistically significant differences between DEB and uncoated balloon angioplasty treatment arms. Study authors reported that primary vessel patency was $97.1 \%$ (95\% confidence interval (CI) 0.91 to 1.00$), 58.8 \%$ (95\% $\mathrm{Cl} 0.44$ to 0.78$)$, and $40.7 \%$ (95\% Cl 0.25 to 0.64 ) in the DEB group, and $97.1 \%$ (95\% $\mathrm{Cl} 0.91$ to 1.00$)$, $31.3 \%$ (95\% $\mathrm{Cl} 0.18$ to 0.52$)$, and $13.4 \%(95 \% \mathrm{Cl} 0.05$ to 0.36$)$ in the uncoated balloon angioplasty group at one, six, and 12 months, respectively. Study authors did not report the number of participants included in the calculations, however, so it is not possible to determine whether these percentages were calculated using an intentionto-treat analysis, nor could we perform a pooled analysis of trial results.

\section{Secondary vessel patency \\ Late lumen loss}

None of the included studies reported late lumen loss (FAIR; ISARPEBIS; PACUBA).

\section{Target lesion revascularization}

One study reported TLR at six and 24 months of follow-up (ISARPEBIS). At six months, participants treated with DEB had lower odds of requiring TLR compared with participants who underwent uncoated balloon angioplasty (Analysis 2.1; odds ratio (OR) 0.05 , $95 \% \mathrm{Cl} 0.00$ to $0.92 ; 70$ participants; 1 study; very low-certainty evidence). Similarly, at 24 months, participants treated with DEB had lower odds of requiring TLR compared with participants who underwent uncoated balloon angioplasty (Analysis 4.1; OR 0.24, $95 \% \mathrm{Cl} 0.08$ to 0.70 ; 70 participants; 1 study).

\section{Freedom from target lesion revascularization}

Two studies reported freedom from TLR at six and 12 months (FAIR; PACUBA). In the FAIR trial, freedom from TLR at six months was reported as $96.4 \% \pm 3 \%$ standard error (SE) for DEB, and as $81 \% \pm$ $5 \%$ SE for uncoated balloon angioplasty $(P=0.0117)$. At 12 months, freedom from TLR was reported as $90.8 \% \pm 6 \%$ SE for DEB and $52.6 \% \pm 10 \%$ SE for uncoated balloon angioplasty $(P<0.0001)$. The PACUBA trial reported freedom from TLR as $88.2 \%(95 \% \mathrm{Cl}$ 0.78 to 0.99$)$ at six months for DEB and $83.8 \%(95 \% \mathrm{Cl} 0.72$ to 0.97 ) for uncoated balloon angioplasty. At 12 months, study authors reported freedom from TLR as $49 \%(95 \% \mathrm{Cl} 0.32$ to 0.75$)$ for DEB and $22.1 \%(95 \% \mathrm{Cl} 0.10$ to 0.47$)$ for uncoated balloon angioplasty. Study authors did not report the number of participants included in the calculations, however, so it is not possible to determine whether these percentages were calculated using an intentionto-treat analysis, nor could we perform a pooled analysis of trial results.

\section{Binary restenosis rate}

Two studies reported binary restenosis at six months (FAIR; ISARPEBIS), and one study reported binary restenosis at 12 months (FAIR). The FAIR trial authors defined binary restenosis as a peak systolic velocity ratio $\geq 2.4$ by duplex ultrasound, and the ISARPEBIS trial authors did not list their criteria for binary restenosis. At six months, participants treated with DEB had significantly lower odds of binary restenosis compared with those treated

Drug-eluting balloon angioplasty versus uncoated balloon angioplasty for the treatment of in-stent restenosis of the femoropopliteal 12

arteries (Review)

Copyright (c) 2019 The Cochrane Collaboration. Published by John Wiley \& Sons, Ltd. 
with uncoated balloon angioplasty (Analysis 2.2; OR 0.28, 95\% $\mathrm{Cl} 0.14$ to 0.56 ; 189 participants; 2 studies; very low-certainty evidence). Similarly, at 12 months, participants treated with DEB had significantly lower odds of binary restenosis compared with those treated with uncoated balloon angioplasty (OR $0.34,95 \% \mathrm{Cl}$ 0.15 to 0.76 ; 119 participants; 1 study).

\section{Death}

All three included studies reported death at six months (FAIR; ISARPEBIS; PACUBA), two studies reported death at 12 months (FAIR; PACUBA), and one study reported death at 24 months (ISAR-PEBIS). The PACUBA study did not report any deaths during the course of the study for participants treated with DEB or uncoated balloon angioplasty. At six months, DEB showed no mortality advantage over uncoated balloon angioplasty (Analysis 2.3; OR 0.93, 95\% $\mathrm{Cl} 0.13$ to $6.71 ; 263$ participants; 3 studies; very low-certainty evidence). Similarly, DEB offered no mortality advantage over uncoated balloon angioplasty at 12 months (Analysis 3.2; OR 0.60, $95 \% \mathrm{Cl} 0.10$ to $3.73 ; 193$ participants; 2 studies) nor at 24 months (Analysis 4.2; OR $7.21,95 \% \mathrm{Cl} 0.36$ to $144.95 ; 70$ participants; 1 study).

\section{Secondary outcomes}

\section{Change in Rutherford category of PAD}

None of the included studies reported the change in category of PAD (FAIR; ISAR-PEBIS; PACUBA). However, the PACUBA trial reported the number of participants who had improvement $\geq$ 1 Rutherford category at one, six, and 12 months, and the FAIR trial reported the number of participants who experienced improvement $\geq 1$ Rutherford category at six months. At one month, DEB showed no advantage over uncoated balloon angioplasty for participants experiencing improvement $\geq 1$ Rutherford category (Analysis 1.1; OR 2.33, 95\% Cl 0.55 to 9.83; 74 participants; 1 study). However, participants treated with DEB had significantly greater odds of experiencing improvement $\geq 1$ Rutherford category at six months (Analysis 2.4; OR $1.81,95 \% \mathrm{Cl} 1.02$ to 3.21 ; 193 participants; 2 studies; very low-certainty evidence) and at 12 months (Analysis 3.3; OR 2.08, 95\% Cl 1.13 to 3.83; 193 participants; 2 studies).

\section{Change in the Society for Vascular Surgery Wound, Ischemia, and Foot Infection (WIfI) stage}

None of the included studies reported change in WIfi stage (FAIR; ISAR-PEBIS; PACUBA).

\section{Change in ankle-brachial index (ABI)}

None of the included studies reported change in ABI (FAIR; ISARPEBIS; PACUBA). Two studies reported $A B I$ at baseline (FAIR; $P A C U B A)$, and one study reported $A B I$ at one month (PACUBA). Both the FAIR and PACUBA studies reported $A B I$ at six and 12 months. The mean $A B I$ at baseline was $0.63 \pm 0.27$ for DEB participants and $0.64 \pm 0.25$ for uncoated balloon angioplasty participants in the FAIR trial, and $0.65 \pm 0.16$ for DEB participants and $0.65 \pm 0.16$ for uncoated balloon angioplasty participants in the PACUBA trial. At one month, the mean $\mathrm{ABI}$ was $0.88 \pm 0.14$ for $\mathrm{DEB}$ participants and $0.9 \pm 0.13$ for uncoated balloon angioplasty participants in the PACUBA trial. At six months, the mean ABI was $0.9 \pm 0.25$ for DEB participants and $0.86 \pm 0.3$ for uncoated balloon angioplasty participants in the FAIR trial, and $0.79 \pm 0.13$ for DEB participants and $0.78 \pm 0.18$ in the PACUBA trial. At 12 months, the mean $A B I$ was $0.86 \pm 0.3$ for DEB participants and $0.9 \pm 0.17$ for uncoated balloon angioplasty participants in the FAIR trial, and $0.79 \pm 0.2$ for DEB participants and $0.84 \pm 0.3$ for uncoated balloon angioplasty participants in the PACUBA trial. The authors of both the FAIR and PACUBA studies did not report the number of participants included in the calculations, however, so it is not possible to determine whether these percentages were calculated using an intentionto-treat analysis, nor could we perform a pooled analysis of trial results.

\section{Change in quality of life (QoL) scores}

None of the included studies reported change in QoL scores (FAIR; ISAR-PEBIS; PACUBA).

\section{Change in functional walking ability}

None of the included studies reported change in functional walking ability (FAIR; ISAR-PEBIS; PACUBA).

\section{Subgroup analysis}

It is not possible to carry out a subgroup analysis, as data were not available from more than two trials for any of the reported outcomes.

\section{Sensitivity analysis}

It is not possible to carry out a sensitivity analysis because of the small number of included studies and the discrepancy in time points at which included studies reported outcomes. Furthermore, none of the included studies were powered to detect clinical endpoints, and several of our pre-specified outcomes were not reported.

\section{IS C U S S I O N}

\section{Summary of main results}

Drug-eluting balloon (DEB) angioplasty was associated with lower odds of requiring target lesion revascularization (TLR) for up to 24 months and lower rates of binary restenosis for up to 12 months when compared with uncoated balloon angioplasty for treatment of symptomatic in-stent restenosis. The drug-eluting balloon was also associated with greater odds of improvement in the Rutherford category of peripheral arterial disease (PAD) for up to 12 months when compared with uncoated balloon angioplasty. We noted no clear difference in amputation or mortality between DEB and uncoated balloon angioplasty at up to 24 months of follow-up. Because of lack of reporting, evidence was insufficient to suggest an advantage for DEB in primary vessel patency; amputation-free survival; late lumen loss; change in Society for Vascular Surgery Wound, Ischemia, and Foot Infection (WIfI) stage; ankle-brachial index (ABI); quality of life (QoL) scores; or functional walking ability when compared with uncoated balloon angioplasty for treatment of in-stent restenosis. Data from the three included trials were also insufficient for performance of subgroup or sensitivity analyses.

\section{Overall completeness and applicability of evidence}

All studies reported on symptomatic in-stent restenosis. All included studies reported a number of anatomical and clinical outcomes that are relevant to practitioners who treat in-stent restenosis. Unfortunately, all included studies had significant limitations (listed in Risk of bias in included studies). All three trials were at high risk of bias due to significant losses of participants to follow-up, lack of blinding, and lack of stratification by type of 
stent implanted and requirement for bailout stenting. Use of TLR as an outcome measure is also potentially problematic, as it is a highly subjective measure that makes it difficult to standardize who will be offered a reintervention. Similarly, change in Rutherford category is a highly subjective measure, and it does not seem that any of the included trials used objective measures, such as treadmill testing, to enable determination of Rutherford category. Most of the participants in the trial did not have evidence of critical limb ischemia, as defined by a Rutherford category $\geq 4$, which is the main indication for many physicians in treating peripheral arterial disease. Furthermore, it is not possible to perform any subgroup analyses nor to exclude any trials from a sensitivity analysis. Finally, the included studies did not assess many of the pre-planned outcomes identified in our study protocol, and some of the outcomes data, such as the ABI, were not amenable to pooled analysis because of the format in which they were reported. Given that we identified at least two ongoing studies (listed in Ongoing studies), we are hopeful that it will become possible someday to carry out a more rigorous examination of the role of DEB in treating in-stent restenosis.

\section{Quality of the evidence}

This review was based on three studies that recruited a relatively small number of participants and were powered primarily to detect anatomical rather than clinical outcomes. However, all analyses in this review are based on data from a single trial or from two trials. As such, caution should be taken in over-interpreting clinical findings, such as the incidence of amputation or change in ABI. Studies used two DEBs with different paclitaxel doses, and two of the three studies were sponsored by a DEB manufacturer (Medtronic). Unfortunately, the included studies did not report most of the outcomes that we identified as important in this review. Finally, all three studies were at high risk of performance, attrition, and other biases, and this negatively impacts the overall quality of the evidence.

\section{See Summary of findings for the main comparison.}

\section{Potential biases in the review process}

We carried out this review according to the principles outlined in the Cochrane guidelines. We encountered difficulty obtaining data from the FAIR and ISAR-PEBIS authors, as well as from authors of the ongoing studies (Copa Cabana; TRC-14004848). Because of the limited number of studies included in this review, we were unable to carry out any subgroup or sensitivity analyses.

\section{Agreements and disagreements with other studies or reviews}

We identified two systematic reviews that examined the role of DEB for in-stent restenosis (Cassese 2017; Wu 2017). Cassese 2017 , the first review, similarly reported superior outcomes with DEB compared with uncoated balloon angioplasty in TLR and binary restenosis, and showed significant improvement in Rutherford category. The Cassese review differs from our review on several points, however. First, the Cassese 2017 review authors chose to include a non-randomized study that we excluded (DEBATE-ISR), along with unpublished data from the Copa Cabana trial, which we have not been able to verify. Furthermore, Cassese 2017 authors did not include ISAR-PEBIS in the analysis. Second, review authors for Cassese 2017 did not specify the time points at which the analysis was carried out, electing instead to report outcomes for the included studies at an unspecified time point. As such, it is not possible to assess whether their reported improvement in TLR, for example, occurred at six or 12 months, because the studies that they included in their analysis reported outcomes at different time points.

The second systematic review also reported superior outcomes with DEB compared with uncoated balloon angioplasty in TLR and binary restenosis, as well as clinical improvement (Wu 2017), which, in our study, we identified as a positive change in Rutherford category. However, Wu 2017 also included DEBATE-ISR in the analysis (which we excluded because it is non-randomized) and did not include data from the ISAR-PEBIS trial.

\section{AUTHORS' CONCLUSIONS}

\section{Implications for practice}

All three included trials were powered to detect anatomical rather than clinically relevant endpoints (FAIR; ISAR-PEBIS; PACUBA). Our analysis showed an advantage for DEB over uncoated balloon angioplasty for treatment of in-stent restenosis for up to two years in some domains. However, the certainty of the evidence is generally very low because of the small number of included studies and the risk of bias in those studies. As such, evidence is insufficient at present to show the superiority of DEB over uncoated balloon angioplasty for treatment of in-stent restenosis. This has important implications for practice, given the increased costs associated with the use of DEBs, which are generally more expensive than uncoated balloon angioplasty catheters.

\section{Implications for research}

To properly evaluate the role of DEBs in treating patients with in-stent restenosis, appropriately powered, independently funded clinical trials are needed to generate rigorous and reliable data that can be used in a future iteration of this review and that answer questions that are clinically relevant to patients. Questions that can be answered in future trials involve the characteristics of stents that would benefit most from DEB angioplasty for in-stent restenosis and the effective dose of paclitaxel that would prevent further restenosis.

\section{ACK N O WLEDGEMENTS}

The review authors would like to thank Drs. Marlene Stewart and Cathryn Broderick for their guidance and expert help in preparing this protocol. 


\section{R E F E R E N C E S}

\section{References to studies included in this review}

FAIR \{published data only (unpublished sought but not used)\} Krankenberg H. FAIR drug eluting balloon vs. PTA for superficial femoral artery in-stent restenosis 12-month results. The Leipzig Interventional Course. Leipzig 28-31 January 2014.

* Krankenberg H, Tübler T, Ingwersen M, Schlüter M, Scheinert D, Blessing E, et al. Drug-coated balloon versus standard balloon for superficial femoral artery in-stent restenosis: the randomized femoral artery in-stent restenosis (FAIR) trial. Circulation 2015;132(23):2230-6.

NCT01305070. Standard balloon angioplasty versus angioplasty with a paclitaxel-eluting balloon for Femoral Artery In-stent Restenosis (FAIR). clinicaltrials.gov/ct2/show/NCT01305070 (date first received February 28, 2011).

ISAR-PEBIS \{published data only (unpublished sought but not used)\}

NCT01083394. Paclitaxel eluting balloon and conventional balloon for in-stent restenosis of the superficial femoral artery (ISAR-PEBIS). clinicaltrials.gov/ct2/show/NCT01083394 (date first received March 9, 2010).

* Ott I, Cassese S, Groha P, Steppich B, Voll F, Hadamitzky M, et al. ISAR-PEBIS (paclitaxel-eluting balloon versus conventional balloon angioplasty for in-stent restenosis of superficial femoral artery): a randomized trial. Journal of the American Heart Association 2017;6(7):pii: e006321.

PACUBA \{published data only (unpublished sought but not used)\}

* Kinstner CM, Lammer J, Willfort-Ehringer A, Matzek W, Gschwandtner M, Javor D, et al. Paclitaxel-eluting balloon versus standard balloon angioplasty in in-stent restenosis of the superficial femoral and proximal popliteal artery: 1-year results of the PACUBA trial. JACC Cardiovascular Interventions 2016;9(13):1386-92.

Kinstner CM, Schoder M, Funovics MA, Willfort-Ehringer A, Gschwandtner M, Ristl R, et al. A monocenter randomized clinical trial of PAClitaxel drUgeluting Balloon versus standard percutaneous transluminal Angioplasty to reduce restenosis in patients with in-stent stenoses in the superficial femoral and proximal popliteal artery (PACUBA I Trial). Cardiovascular and Interventional Radiology 2015;38(3 Suppl 1):S288.

NCT01247402. Paclitaxel balloon versus standard balloon in in-stent restenoses of the superficial femoral artery (PACUBA I Trial) (PACUBA 1). clinicaltrials.gov/ct2/show/NCT01247402 (date first received November 24, 2010).

\section{References to studies excluded from this review}

Bosiers 2015 \{published data only\}

Bosiers M, Deloose K, Callaert J, Verbist J, Hendriks J, Lauwers $P$, et al. Superiority of stent-grafts for in-stent restenosis in the superficial femoral artery: twelve-month results from a multicenter randomized trial. Journal of Endovascular Therapy 2015;22(1):1-10.

\section{ConSeQuent \{published data only\}}

Albrecht T, Meyer D, Müller-Hülsbeck S, Ott I, Redlich U, Ricke J, et al. Preliminary angiographic and clinical 6-month results of the CONSEQUENT trial. The Leipig Interventional Course. Leipzig 26-29 January 2016.

NCT01970579. Clinical trial on peripheral arteries treated with SeQuent ${ }^{\circledR}$ Please P Paclitaxel coated balloon catheter (ConSeQuent). clinicaltrials.gov/ct2/show/NCT01970579 (date first received October 28, 2013).

\section{DEBATE-ISR \{published data only\}}

Liistro F, Angioli P, Porto I, Ricci L, Ducci K, Grotti S, et al. Paclitaxel-eluting balloon vs. standard angioplasty to reduce recurrent restenosis in diabetic patients with in-stent restenosis of the superficial femoral and proximal popliteal arteries: the DEBATE-ISR study. Journal of Endovascular Therapy 2014;21(1):1-8.

\section{EXCITE ISR \{published data only\}}

* Dippel EJ, Makam P, Kovach R, George JC, Patlola R, Metzger DC, et al. Randomized controlled study of excimer laser atherectomy for treatment of femoropopliteal instent restenosis: initial results from the EXCITE ISR trial (EXCImer Laser Randomized Controlled Study for Treatment of FemoropopliTEal In-Stent Restenosis). JACC.Cardiovascular interventions 2015;8:92-101.

Dippel EJ, Makam P, Kovach RC, George JC, et al. EXCITE ISR: a prospective, randomized controlled trial of Excimer laser atherectomy vs balloon angioplasty for the treatment of femoropopliteal in-stent restenosis. Journal of the American College of Cardiology 2014; Vol. 64, issue Suppl 11.

NCT01330628. Randomized study of laser and balloon angioplasty versus balloon angioplasty to treat peripheral in-stent restenosis (EXCITE ISR). clinicaltrials.gov/ct2/show/ NCT01330628 (date first received April 7, 2011).

\section{NCT00481780 \{published data only\}}

NCT00481780. Conventional balloon angioplasty vs. cutting balloon angioplasty for treatment of femoropopliteal artery in-stent restenosis - a randomized controlled pilot trial. clinicaltrials.gov/ct2/show/NCT00481780 (date first received June 4, 2007).

\section{NCT02832024 \{published data only\}}

NCT02832024. Clinical study of stent versus direct atherectomy versus angioplasty to treat lower limb in-stent restenosis. clinicaltrials.gov/ct2/show/NCT02832024 (date first received July 13, 2016).

\section{RELINE \{published data only\}}

Deloose K. RELINE randomized clinical trial: Viabahn covered stents vs PTA. The Leipzig Interventional Course. Leipzig 28-31 January 2014.

NCT01108861. GORE VIABAHN ${ }^{\circledR}$ versus plain old balloon angioplasty (POBA) for superficial femoral artery (SFA) in-stent

Drug-eluting balloon angioplasty versus uncoated balloon angioplasty for the treatment of in-stent restenosis of the femoropopliteal 
restenosis (RELINE). clinicaltrials.gov/ct2/show/NCT01108861 (date first received April 22, 2010).

\section{References to ongoing studies}

Copa Cabana \{published data only (unpublished sought but not used)\}

NCT01594684. Cotavance ${ }^{\mathrm{TM}}$ paclitaxel-coated balloon versus uncoated balloon angioplasty for treatment of in-stent restenosis in SFA and popliteal arteries. clinicaltrials.gov/ct2/ show/results/NCT01594684 (date first received May 9, 2012).

TRC-14004848 \{published data only (unpublished sought but not used)\}

ChiCTR-TRC-14004848. Drug-eluting balloon versus standard balloon angioplasty for the treatment of in-stent restenosis in the femoral-popliteal artery: a randomized, controlled prospective study. chictr.org.cn/hvshowproject.aspx?id=11037 (date first received May 1, 2015).

\section{Additional references}

\section{Alfonso 2014}

Alfonso F, Byrne RA, Rivero F, Kastrati A. Current treatment of instent restenosis. Journal of the American College of Cardiology 2014;63(24):2659-73.

\section{Ali 2012}

Ali FN, Carman TL. Medical management for chronic atherosclerotic peripheral arterial disease. Drugs 2012;72(16):2073-85.

\section{Atkins 2004}

Atkins D, Best D, Briss PA, Eccles M, Falck-Ytter Y, Flottorp S, et al. Grading quality of evidence and strength of recommendations. BMJ 2004;328(7454):1490-4.

\section{Canaud 2014}

Canaud L, Ozdemir BA, Belli AM, Loftus IM, Thompson MM, Hinchliffe RJ. Infrainguinal angioplasty with drug-eluting stents and balloons. Journal of Vascular Surgery 2014;59(6):1721-36.

\section{Cassese 2017}

Cassese S, Ndrepepa G, Kufner S, Byrne RA, Giacoppo D, Ott I, et al. Drug-coated balloon angioplasty for in-stent restenosis of femoropopliteal arteries: a meta-analysis. Eurolntervention 2017;13(4):483-9.

\section{Conte 2015}

Conte MS, Pomposelli FB, Clair DG, Geraghty PJ, McKinsey JF, Mills JL, et al. Society for Vascular Surgery practice guidelines for atherosclerotic occlusive disease of the lower extremities: management of asymptomatic disease and claudication. Journal of Vascular Surgery 2015;61(3 Suppl):2S-41S.

\section{Criqui 2015}

Criqui $\mathrm{MH}$, Aboyans V. Epidemiology of peripheral artery disease. Circulation Research 2015;116(9):1509-26.

\section{Deeks 2011}

Deeks JJ, Higgins JP, Altman DG, editor(s). Chapter 9: Analysing data and undertaking meta-analyses. In: Higgins JP, Green S, editor(s). Cochrane Handbook for Systematic Reviews of Interventions Version 5.1.0 (updated March 2011). The Cochrane Collaboration, 2011. Available from handbook.cochrane.org.

\section{Higgins 2003}

Higgins JPT, Thompson SG, Deeks JJ, Altman DG. Measuring inconsistency in meta-analyses. BMJ 2003;327:557-60.

\section{Higgins 2011}

Higgins JP, Altman DG, Sterne JA, editor(s). Chapter 8: Assessing risk of bias in included studies. In: Higgins JP, Green S, editor(s). Cochrane Handbook for Systematic Reviews of Interventions Version 5.1.0 (updated March 2011). The Cochrane Collaboration, 2011. Available from handbook.cochrane.org.

\section{Indermuehle 2013}

Indermuehle A, Bahl R, Lansky AJ, Froehlich GM, Knapp G, Timmis A, et al. Drug-eluting balloon angioplasty for instent restenosis: a systematic review and meta-analysis of randomised controlled trials. Heart 2013;99(5):327-33.

\section{Marupudi 2007}

Marupudi NI, Han JE, Li KW, Renard VM, Tyler BM, Brem H. Paclitaxel: a review of adverse toxicities and novel delivery strategies. Expert Opinion on Drug Safety 2007;6(5):609-21.

\section{McDermott 2001}

McDermott MM, Greenland P, Liu K, Guralnik JM, Criqui MH, Dolan NC, et al. Leg symptoms in peripheral arterial disease: associated clinical characteristics and functional impairment. Journal of the American Medical Association 2001;286(13):1599-606.

\section{Mills 2014}

Mills JL, Conte MS, Armstrong DG, Pomposelli FB, Schanzer A, Sidawy AN, et al. The Society for Vascular Surgery lower extremity threatened limb classification system: risk stratification based on Wound, Ischemia, and foot Infection (WIfI). Journal of Vascular Surgery 2014;59(1):220-34.

\section{OHTAC 2010}

Ontario Health Technology Advisory Committee. Stenting for peripheral artery disease of the lower extremities: an evidencebased analysis. Ontario Health Technology Assessment Series 2010;10(18):1-88

\section{Review Manager 2014 [Computer program]}

The Nordic Cochrane Centre, The Cochrane Collaboration. Review Manager 5 (RevMan 5). Version 5.3. Copenhagen: The Nordic Cochrane Centre, The Cochrane Collaboration, 2014.

\section{Schünemann 2011}

Schünemann HJ, Oxman AD, Higgins JP, Vist GE, Glasziou P, Guyatt GH. Chapter 11: Presenting results and 'Summary of findings' tables. In: Higgins JP, Green S, editor(s), Cochrane Handbook for Systematic Reviews of Interventions Version 5.1.0 (updated March 2011). The Cochrane Collaboration, 2011. Available from handbook.cochrane.org. 


\section{Schünemann 2011b}

Schünemann HJ, Oxman AD, Vist GE, Higgins JP, Deeks JJ, Glasziou P, et al. Chapter 12: Interpreting results and drawing conclusions. In: Higgins JP, Green S, editor(s), Cochrane Handbook for Systematic Reviews of Interventions Version 5.1.0 (updated March 2011). The Cochrane Collaboration, 2011. Available from handbook.cochrane.org.

\section{Singh 2014}

Singh GD, Armstrong EJ, Laird JR. Femoropopliteal instent restenosis: current treatment strategies. Journal of Cardiovascular Surgery 2014;55(3):325-33.

\section{Stary 1994}

Stary H, Chandler AB, Glagov S, Guyton JR, Insull W, Rosenfeld ME, et al. A definition of initial, fatty streak, and intermediate lesions of atherosclerosis. A report from the Committee on Vascular Lesions of the Council on Arteriosclerosis, American Heart Association. Circulation 1994;89(5):2462-78.

\section{Sterne 2011}

Sterne JAC, Egger M, Moher D, editor(s). Chapter 10: Addressing reporting biases. In: Higgins JP, Green S, editor(s). Cochrane

\section{CHARACTERISTICS OF STUDIES}

Characteristics of included studies [ordered by study ID]
Handbook for Systematic Reviews of Interventions. Version 5.1.0 (updated March 2011). The Cochrane Collaboration, 2011. Available from handbook.cochrane.org.

\section{Waksman 2009}

Waksman R, Pakala R. Drug-eluting balloon: the comeback kid?. Circulation Cardiovascular Interventions 2009;2(4):352-8.

\section{Wu 2017}

Wu R, Li Z, Wang M, Chang G, Yao C, Wang S. Paclitaxel-coated versus uncoated balloon angioplasty for femoropopliteal artery in-stent restenosis. International Journal of Surgery 2017;42:72-82.

\section{References to other published versions of this review}

\section{Kayssi 2017}

Kayssi A, Al-Jundi W, Papia G, Kucey DS, Forbes T, Rajan DK, et al. Drug-eluting balloon angioplasty versus uncoated balloon angioplasty for the treatment of in-stent restenosis of the femoropopliteal arteries. Cochrane Database of Systematic Reviews 2017, Issue 1. [DOI: 10.1002/14651858.CD012510]

* Indicates the major publication for the study

\section{FAIR}

Methods

Study design: prospective, multicenter trial

Method of randomization: block randomization (1:1 uncoated balloon angioplasty or DEB)

Blinding: non-blinded

Exclusions post randomization: none

Losses to follow-up: 6 months: DEB 16.1\%, uncoated balloon angioplasty $17.5 \% ; 12$ months: DEB 29\%, uncoated balloon angioplasty $29.8 \%$

Study enrollment period: January 2010 to November 2012

Cross-over: none

Participants Country: Germany

Setting: 5 vascular centers

Number of participants: 119

Age (mean): DEB $69 \pm 8$ years, uncoated balloon angioplasty $67 \pm 9$ years

Gender: DEB 53.2\% male, uncoated balloon angioplasty $70.2 \%$ male

Rutherford class: 1: DEB 43.5\%, uncoated balloon angioplasty 147.4\%; 2: DEB 51.6\%, uncoated balloon angioplasty 42.1\%; 4: DEB 1.6\%, uncoated balloon angioplasty $10.5 \%$; 5: DEB 3.2\%, uncoated balloon angioplasty $0 \%$ 
FAIR (Continued)

Inclusion criteria: superficial femoral artery in-stent restenosis of up to $20 \mathrm{~cm}$ in length; diameter stenosis at least $70 \%$ by duplex ultrasound (DUS); popliteal artery and 1 of infrapopliteal vessels patent for sustained distal runoff; chronic limb ischemia (Rutherford 2 to 4 )

Exclusion criteria: untreated ipsilateral iliac artery stenosis, ongoing dialysis treatment, treatment with oral anticoagulants other than antiplatelet agents

Uncoated balloon angioplasty: 57 participants
Uncoated balloon angioplasty device: Admiral Xtreme (Medtronic)
DEB: 62 participants
DEB device: IN.PACT Admiral (Medtronic)
Drug used: paclitaxel $3.5 \mathrm{mcg} / \mathrm{mm}^{2}$
Vessel treated: superficial femoral artery
Anticoagulation/antiplatelets: preoperatively: pre-medication with aspirin $100 \mathrm{mg} / \mathrm{d}$ and clopidogrel
$75 \mathrm{mg} / \mathrm{d}$ for at least 10 days. Participants not on this regimen were given a bolus of $500 \mathrm{mg}$ aspirin in-
travenously and clopidogrel $600 \mathrm{mg}$ orally before or immediately after the intervention. Postoperative-
ly: participants received aspirin $100 \mathrm{mg} / \mathrm{d}$ indefinitely and clopidogrel $75 \mathrm{mg} / \mathrm{d}$ for at least $6 \mathrm{months}$

Outcomes

Primary: incidence of binary recurrent in-stent restenosis at 6 months as assessed by ultrasound (peak systolic velocity ratio $\geq 2.4$ )

\section{Secondary:}

- Procedural endpoints: primary angiographic success (successful access and deployment of the device with $\leq 50 \%$ diameter residual stenosis without bailout procedures), cumulative incidence of binary recurrent restenosis at 12 months, Kaplan-Meier estimate of freedom from target lesion revascularization based on recurrent restenosis $\geq 50 \%$ reocclusion and clinical signs through 6 - and 12 -month follow-up (not including procedural bailout)

- Hemodynamic endpoints: ankle brachial index at 6 and 12 months and immediate and sustained hemodynamic success (ankle brachial index improvement $\geq 0.15$ from baseline to discharge and to 6 and 12 months without the need for target lesion revascularization)

- Clinical endpoints: sustained clinical improvement by $\geq 1$ Rutherford category relative and absolute claudication distance at 6 and 12 months; major adverse vascular events, defined as all-cause death, myocardial infarction, major amputation, major bleeding, and thrombosis or surgical intervention related to the target limb

\begin{tabular}{|c|c|c|}
\hline Notes & \multicolumn{2}{|c|}{$\begin{array}{l}\text { Follow-up: } 6 \text { and } 12 \text { months } \\
\text { Sponsor: Medtronic } \\
\text { Strengths/limitations: } \\
\text { - Strengths: used widely available devices, simple and generalizable study design } \\
\text { - Limitations: significant } 12 \text {-month loss to follow-up ( } 29 \% \text { and } 29.8 \% \text { in the uncoated balloon angio- } \\
\text { plasty and DEB arms, respectively), not powered to detect clinical outcomes, industry sponsored }\end{array}$} \\
\hline \multicolumn{3}{|l|}{ Risk of bias } \\
\hline Bias & Authors' judgement & Support for judgement \\
\hline $\begin{array}{l}\text { Random sequence genera- } \\
\text { tion (selection bias) }\end{array}$ & Low risk & $\begin{array}{l}\text { Block randomization was used in the study with a block size of } 10 \text { and an allo- } \\
\text { cation ratio of } 1: 1\end{array}$ \\
\hline
\end{tabular}


FAIR (Continued)

$\begin{array}{ll}\begin{array}{l}\text { Allocation concealment } \\ \text { (selection bias) }\end{array} & \text { Low risk } \\ \end{array}$

Blinding of participants High risk
and personnel (perfor-
mance bias)

$\begin{array}{ll}\begin{array}{l}\text { Blinding of outcome as- } \\ \text { sessment (detection bias) }\end{array} \quad \text { Unclear risk } & \text { Clinical status was evaluated before the procedure and again at } 6 \text { and } 12 \\ \text { months, but whether the outcome assessment was blinded remains unclear }\end{array}$

Incomplete outcome data High risk (attrition bias)

All outcomes

All outcomes

Selective reporting (re- Low risk porting bias)

\begin{tabular}{ll}
\hline Other bias & High risk \\
& $\begin{array}{l}\text { Study authors did not specify the types of stents that were treated in this } \\
\text { study. It is possible that particular types of stents (e.g. covered vs bare-metal) } \\
\text { might respond differently to treatment. Furthermore, more uncoated balloon } \\
\text { angioplasty participants received bailout stenting compared with DEB partici- } \\
\text { pants (4 vs } 1 \text { participant) }\end{array}$ \\
\hline
\end{tabular}
Approximately one-third of trial participants were lost to follow-up at 12
months

\section{ISAR-PEBIS}

Study design: prospective, randomized, active-controlled trial
Method of randomization: $1: 1$ uncoated balloon angioplasty or DEB
Blinding: single (outcomes assessor)
Exclusions post randomization: none
Losses to follow-up: 6 months: DEB 3\%, uncoated balloon angioplasty $3 \% ; 24$ months: DEB $22 \%$, un-
coated balloon angioplasty $15 \%$

Study enrollment period: April 2010 to December 2013

Cross-over: none

Participants Country: Germany

Setting: 2 vascular centers

Number of participants: 70

Age (mean): DEB $70 \pm 10$ years, uncoated balloon angioplasty $68 \pm 10$ years

Gender: DEB 67\% male, uncoated balloon angioplasty $70 \%$ male

Rutherford class: 2: DEB 3\%, uncoated balloon angioplasty 0\%; 3: DEB 94\%, uncoated balloon angioplasty $97 \%$; 4: DEB and uncoated balloon angioplasty $0 \%$; 5: DEB 3\%, uncoated balloon angioplasty $3 \%$

\section{Inclusion criteria:}

- Symptomatic $\geq 70 \%$ in-stent restenosis of the superficial femoral artery (Rutherford stage 2 to 6 ) 
-Written, informed consent by participant or her/his legally authorized representative for participation in the study

\section{Exclusion criteria:}

- Acute ischemia and/or acute thrombosis of the superficial femoral artery

- Untreated ipsilateral iliac artery stenosis $>70 \%$

- Not at least 1 vessel runoff

- Popliteal involvement with stenosis $>70 \%$

- Severe renal insufficiency (glomerular filtration rate $<30 \mathrm{~mL} / \mathrm{min} / \mathrm{m}^{2}$ )

- Malignancies or other comorbid conditions (e.g. severe liver, renal, and pancreatic disease) with life expectancy less than 12 months, or that may result in protocol non-compliance

- Pregnancy (present, suspected, or planned) or positive pregnancy test

- Previous enrollment in this trial

- Inability of the patient to fully cooperate with the study protocol

Interventions

\section{Uncoated balloon angioplasty: 34}

Uncoated balloon angioplasty device: Pacific Xtreme (Medtronic)

DEB: 36

DEB device: In.Pact Admiral (Medtronic)

Drug used: paclitaxel $3.5 \mathrm{mcg} / \mathrm{mm}^{2}$

Vessel treated: superficial femoral artery

Anticoagulation/antiplatelets: loading dose of clopidogrel $(600 \mathrm{mg})$ was administered to all clopidogrel-naive participants before angiography. Immediately after the decision was made to perform the intervention, participants were given $500 \mathrm{mg}$ aspirin intravenously and $5000 \mathrm{U}$ heparin intra-arterially. After the intervention, all participants received aspirin $100 \mathrm{mg}$ per day indefinitely and clopidogrel 75 mg per day for at least 6 months

Pre-dilation before DEB: yes, pre-dilation was performed in both study groups with a standard balloon catheter inflated for 2 minutes with a vessel:balloon diameter ratio of 1:1

\section{Secondary:}

- All-cause mortality

- Major adverse peripheral events (MAPEs) defined as acute thrombosis of SFA or ipsilateral amputation or revascularization

- Time to onset of any of MAPE

- Binary restenosis rate

- Percentage diameter stenosis in duplex ultrasound

Follow-up: 6 and 24 months

Sponsor: investigator-initiated trial

Strengths/limitations:

Drug-eluting balloon angioplasty versus uncoated balloon angioplasty for the treatment of in-stent restenosis of the femoropopliteal 
- Strengths: used widely available devices, simple and generalizable study design

- Limitations: significant loss to follow-up for primary outcome (25\% of DEB and $21 \%$ of uncoated bal-

loon angioplasty participants), not powered to detect clinical outcomes

\section{Risk of bias}

\begin{tabular}{lll}
\hline Bias & Authors' judgement & Support for judgement \\
\hline $\begin{array}{l}\text { Random sequence genera- } \\
\text { tion (selection bias) }\end{array}$ & Low risk & $\begin{array}{l}\text { A computer sequence was generated after the decision was made to proceed } \\
\text { with an intervention }\end{array}$ \\
\hline $\begin{array}{l}\text { Allocation concealment } \\
\text { (selection bias) }\end{array}$ & Low risk & Allocation to treatments was accomplished by using sealed, opaque envelopes \\
\hline $\begin{array}{l}\text { Blinding of participants } \\
\text { and personnel (perfor- } \\
\text { mance bias) }\end{array}$ & High risk & Study operators were non-blinded \\
All outcomes & & \\
\hline
\end{tabular}

\begin{tabular}{lll}
\hline $\begin{array}{l}\text { Blinding of outcome as- } \\
\text { sessment (detection bias) } \\
\text { All outcomes }\end{array}$ & Low risk & Outcomes assessor was blinded \\
\hline $\begin{array}{l}\text { Incomplete outcome data } \\
\text { (attrition bias) } \\
\text { All outcomes }\end{array}$ & High risk & $\begin{array}{l}\text { A significant number of participants (25\% of DEB and 21\% of uncoated balloon } \\
\text { angioplasty participants) did not undergo primary endpoint testing (angiogra- } \\
\text { phy) }\end{array}$ \\
\hline $\begin{array}{l}\text { Selective reporting (re- } \\
\text { porting bias) }\end{array}$ & Low risk & All outcomes specified in the study protocol were reported \\
\hline $\begin{array}{l}\text { Other bias } \\
\text { High risk }\end{array}$ & $\begin{array}{l}\text { Study authors did not specify the types of stents that were treated in this } \\
\text { study. It is possible that particular types of stents (e.g. covered vs bare-metal) } \\
\text { might respond differently to treatment. Furthermore, more uncoated balloon } \\
\text { angioplasty participants received bailout stenting compared with DEB partici- } \\
\text { pants (26\% vs } 6 \% \text { of participants; } P=0.02)\end{array}$ \\
\hline
\end{tabular}

\section{PACUBA}

Study design: prospective, dual-center trial
Method of randomization: $1: 1$ randomization via computer-generated sequences
Blinding: single-blinded
Exclusions post randomization: 1 participant in the uncoated balloon angioplasty group because of
lack of technical success due to vessel calcification

Losses to follow-up: 1 month: DEB 3\%, uncoated balloon angioplasty 3\%; 6 months: DEB 3\%, uncoated balloon angioplasty $8 \% ; 12$ months: DEB $23 \%$, uncoated balloon angioplasty $15 \%$

Study enrollment period: November 2010 to December 2012

Cross-over: none

$\begin{array}{ll}\text { Participants } & \text { Country: Austria } \\ & \text { Setting: } 2 \text { academic vascular centers }\end{array}$

Drug-eluting balloon angioplasty versus uncoated balloon angioplasty for the treatment of in-stent restenosis of the femoropopliteal 
PACUBA (Continued)

\section{Number of participants: 74}

Age (mean): DEB $68 \pm 9$ years, uncoated balloon angioplasty $68 \pm 0.4$ years

Gender: DEB 57\% male, uncoated balloon angioplasty 59\% male

Rutherford class: 2: DEB 9\%, uncoated balloon angioplasty 21\%; 3: DEB 91\%, uncoated balloon angioplasty $79 \%$

Results show more Tosaka class I lesions in the DEB group (23\%) compared with the uncoated balloon angioplasty group (5\%), and more Tosaka class II lesions in the uncoated balloon angioplasty group $(67 \%)$ compared with the DEB group (46\%). Similarly, study authors reported more TASC A lesions in the DEB group (23\%) compared with the uncoated balloon angioplasty group (5\%), and more TASC B and $\mathrm{C}$ lesions in the uncoated balloon angioplasty group (36\% and $26 \%$, respectively) compared with the uncoated balloon angioplasty group ( $23 \%$ and $14 \%$, respectively)

\section{Inclusion criteria:}

- Age $>50$ years

- Patient legally authorized to provide written informed consent

- Patient willing and likely to comply with the follow-up schedule

- Patient symptomatic Rutherford-Becker 2 to 5 (Fontaine II to IV)

- In-stent restenosis in the superficial femoral artery and the P1 segment of the popliteal artery

- Tibial runoff of at least 1 artery, which, however, may be stenotic but amenable to PTA

\section{Exclusion criteria:}

- Unable to give informed consent

- Enrolled in another study with any investigational drug or device

- Major surgical procedures (not including minor amputations) within 30 days before this study or planned within 30 days of entry into this study

- Pregnancy

- Any known allergy, hypersensitivity or intolerance to radiological contrast media, ASA, clopidogrel or ticlopidine, or paclitaxel

- Life expectancy less than 1 year

DEB: 35 participants

DEB device: FREEWAY 0.035 DEB (Eurocor)

Drug used: paclitaxel $3 \mathrm{mcg} / \mathrm{mm}^{2}$

Vessels treated: superficial femoral artery and P1 segment of the popliteal artery

Anticoagulation/antiplatelets: aspirin and clopidogrel given at least 1 day before the intervention. Otherwise a loading dose of clopidogrel $300 \mathrm{mg}$ was given during the intervention. All participants received aspirin $100 \mathrm{mg} / \mathrm{d}$ indefinitely and clopidogrel $75 \mathrm{mg} / \mathrm{d}$ for 3 months post intervention

Pre-dilation before DEB: yes 
PACUBA (Continued)

- Primary patency at 6-month follow-up, defined as $<50 \%$ diameter stenosis as demonstrated by duplex ultrasound and CT angiogram in the absence of clinically driven target lesion revascularization during follow-up

- Clinically driven target lesion revascularization defined as reintervention of the target lesion due to presence of a symptomatic $>50 \%$ diameter stenosis

Secondary: severe adverse events

Notes

Follow-up: clinical evaluation at 1, 6, and 12 months follow-up after the index procedure. Duplex ultrasound performed at 24 hours, 6 months, and 12 months, and CT angiogram at 12 months after the index procedure

Sponsor: investigator-initiated trial

\section{Strengths/limitations:}

- Strengths: not industry sponsored

- Limitations: main limitation is that no participants with critical limb ischemia (Rutherford class 4 or greater) were included

\section{Risk of bias}

\begin{tabular}{|c|c|c|}
\hline Bias & Authors' judgement & Support for judgement \\
\hline $\begin{array}{l}\text { Random sequence genera- } \\
\text { tion (selection bias) }\end{array}$ & Low risk & Trial was randomized 1:1 via computer-generated sequences \\
\hline $\begin{array}{l}\text { Allocation concealment } \\
\text { (selection bias) }\end{array}$ & Low risk & Allocation to treatments was accomplished by using sealed envelopes \\
\hline $\begin{array}{l}\text { Blinding of participants } \\
\text { and personnel (perfor- } \\
\text { mance bias) } \\
\text { All outcomes }\end{array}$ & High risk & Study design was single-blinded (outcomes assessor) \\
\hline $\begin{array}{l}\text { Blinding of outcome as- } \\
\text { sessment (detection bias) } \\
\text { All outcomes }\end{array}$ & Low risk & Core laboratory adjudication was blinded \\
\hline $\begin{array}{l}\text { Incomplete outcome data } \\
\text { (attrition bias) } \\
\text { All outcomes }\end{array}$ & High risk & $\begin{array}{l}\text { Eight participants in the DEB group ( } 23 \% \text { ) and } 6 \text { in the uncoated balloon angio- } \\
\text { plasty group (15\%) were lost to follow-up }\end{array}$ \\
\hline $\begin{array}{l}\text { Selective reporting (re- } \\
\text { porting bias) }\end{array}$ & Low risk & All outcomes specified in the study protocol were reported \\
\hline Other bias & High risk & $\begin{array}{l}\text { Study authors did not specify the types of stents that were treated in this } \\
\text { study. It is possible that particular types of stents (e.g. covered vs bare-metal) } \\
\text { might respond differently to treatment. Furthermore, more DEB participants } \\
\text { received bailout stenting compared with uncoated balloon angioplasty partic- } \\
\text { ipants ( } 4 \text { vs } 1 \text { participants). No statistical analysis was performed to compare } \\
\text { the study population's baseline characteristics, but the difference in the num- } \\
\text { ber of Tosaca and TASC lesions between DEB and uncoated balloon angioplas- } \\
\text { ty groups suggests that DEB lesions were less severe }\end{array}$ \\
\hline
\end{tabular}

ASA: aspirin.

$\mathrm{CT}$ : computed tomography.

DEB: drug-eluting balloon.

Drug-eluting balloon angioplasty versus uncoated balloon angioplasty for the treatment of in-stent restenosis of the femoropopliteal 
DUS: duplex ultrasound.

MAPE: major adverse peripheral event.

PTA: percutaneous transluminal angioplasty.

SFA: superficial femoral artery.

Characteristics of excluded studies [ordered by study ID]

\begin{tabular}{|c|c|}
\hline Study & Reason for exclusion \\
\hline Bosiers 2015 & $\begin{array}{l}\text { Randomized controlled trial of covered stent deployment vs balloon angioplasty alone for } \\
\text { femoropopliteal artery in-stent restenosis. No use of drug-eluting balloon technologies in the inter- } \\
\text { ventional arm }\end{array}$ \\
\hline ConSeQuent & $\begin{array}{l}\text { Randomized controlled trial of DEB vs uncoated balloon angioplasty for native femoropopliteal } \\
\text { artery restenosis rather than in-stent restenosis }\end{array}$ \\
\hline DEBATE-ISR & $\begin{array}{l}\text { Non-randomized study of DEB in a prospective cohort of patients presenting with femoropopliteal } \\
\text { artery in-stent restenosis vs an historical cohort of patients who received uncoated balloon angio- } \\
\text { plasty for in-stent restenosis }\end{array}$ \\
\hline EXCITE ISR & $\begin{array}{l}\text { Randomized controlled trial of laser and balloon angioplasty vs balloon angioplasty alone for } \\
\text { femoropopliteal artery in-stent restenosis. No use of drug-eluting balloon technologies in the inter- } \\
\text { ventional arm }\end{array}$ \\
\hline NСT00481780 & $\begin{array}{l}\text { Randomized controlled trial of cutting balloon angioplasty vs balloon angioplasty alone for } \\
\text { femoropopliteal artery in-stent restenosis. No use of drug-eluting balloon technologies in the inter- } \\
\text { ventional arm }\end{array}$ \\
\hline NCT02832024 & $\begin{array}{l}\text { Randomized controlled trial of stent deployment vs atherectomy vs balloon angioplasty alone for } \\
\text { femoropopliteal artery in-stent restenosis. No use of drug-eluting balloon technologies in the inter- } \\
\text { ventional arm }\end{array}$ \\
\hline RELINE & $\begin{array}{l}\text { Randomized controlled trial of covered stent deployment vs balloon angioplasty alone for } \\
\text { femoropopliteal artery in-stent restenosis. No use of drug-eluting balloon technologies in the inter- } \\
\text { ventional arm }\end{array}$ \\
\hline
\end{tabular}

DEB: drug-eluting balloon.

Characteristics of ongoing studies [ordered by study ID]

\section{Copa Cabana}

Trial name or title Cotavance ${ }^{\mathrm{TM}}$ paclitaxel-coated balloon versus uncoated balloon angioplasty for treatment of instent restenosis in SFA and popliteal arteries

Methods Study design: randomized controlled trial

Participants Country: Germany

Setting: 6 academic centers in Germany

Number of participants: 112

\section{Inclusion criteria:}

- In-stent restenosis or reocclusion at the target lesion due to an interventional treatment $>3$ months before enrollment 
- Evidence of $\geq 70 \%$ stenosis or occlusion in the SFA and/or popliteal that is 3 to $27 \mathrm{~cm}$ long and is confirmed by angiography

- Reference vessel diameter of 3 to $7 \mathrm{~mm}$ (reference vessel diameter $=$ normal vessel diameter $1 \mathrm{~cm}$ proximal of the index lesion)

- Patients (men and women) with PAD disease category 2 to 5 according to Rutherford classification

- Patient eligible for an operative vascular intervention in case of complications during the procedure

- Female patients of childbearing potential with negative pregnancy test 7 days before the time of intervention

- Fully informed and signed consent obtained from each patient

- Patient willing and able to continue study participation after study procedure to ensure completion of all procedures and observations required by the protocol

- Patient with evidence of at least 1 runoff vessel who does not also require treatment for significant ( $\geq 50 \%$ stenosis or occlusion) stenosis during the index procedure to the ankle/foot of the limb to be treated. Treatment of infrapopliteal lesions must be staged at least 30 days before or after the index procedure

- If restenosis occurs in both arms within 30 days or later (re-restenosis) - treatment with 2 drugeluting balloons at the same location (double dose)

\section{Exclusion criteria:}

- More than 2 lesions in the target vessel requiring treatment (if the distance between 2 lesions is less than $2 \mathrm{~cm}$, the lesions should be counted as 1 lesion). The second lesion should also be treated with coated or uncoated balloons (according to randomization)

- Guidewire cannot cross lesion and/or an intentional subintimal approach in the stented lesion is required

- Stent fracture grade 2 to 4

- Inflow lesion (proximal to the study lesion) with flow limitation not successfully treated before treatment of the study lesion

- Acute thrombosis of the study lesion requiring lysis or thrombectomy before treatment of the study lesion

- Acutely occurring symptoms with a lysis or an operation as a therapeutic option within the last 6 weeks within the study limb

- Potential loss of leg due to critical or acute ischemia

- No patent distal runoff vessel

- Aneurysm in the blood vessel intended for intervention in this study

- Blood platelet count $<100,000 / \mathrm{mm}^{3}$ or $>700,000 / \mathrm{mm}^{3}$, leukocyte count $<3000 / \mathrm{mm}^{3}$

- Contraindication to anticoagulation or any antiplatelet agent (e.g. aspirin, heparin, clopidogrel, ticlopidine, abciximab), or to paclitaxel

- Known intolerance or contraindication (e.g. severe hepatic (with ALAT and/or ASAT > 3 times the normal reference range) or renal (creatinine $>1.1 \mathrm{mg} / \mathrm{dL}$ in women and $>1.5 \mathrm{mg} / \mathrm{dL}$ in $\mathrm{men}$ ) to contrast agents, which cannot be adequately pre-treated (e.g. with adequate hydration)

- Severe illnesses such as cancer, liver or renal disease, myocardial insufficiency leading to protocol deviation, and/or reduced life expectancy of less than 2 years

- Manifesting hyperthyreosis

Drug-eluting balloon angioplasty versus uncoated balloon angioplasty for the treatment of in-stent restenosis of the femoropopliteal 
Copa Cabana (Continued)

- Latent hyperthyreosis without adequate therapy (e.g. previous blocking with Natriumperchlorat (Irenat))

- Significant gastrointestinal hemorrhage within 6 months before study participation

- History of hemorrhagic diathesis or coagulopathy or rejection of blood transfusion

- Medical reason against double antiplatelet therapy in anticoagulated patient (e.g. receiving Coumadin)

- Any severe medical condition that might interfere with interpretation of the data or might result in unacceptable risk for the patient's participation in the study, according to the judgment of the clinical investigator

- Female patient who is pregnant or lactating

- Patient younger than 18 years of age

- Participation in another clinical study up to 30 days before study entry

- Previous participation in the same trial (only 1 leg can be treated within the study)
Uncoated balloon angioplasty device: unspecified

DEB device: Cotavance DEB (Medrad)

Vessels treated: femoropopliteal arteries

\section{Outcomes}

Primary: late lumen loss at 6 months

\section{Secondary:}

- Procedural success

- Target lesion revascularization

- Target vessel revascularization

- Binary restenosis rate

- Rutherford category

- Ankle-brachial index

- Late lumen loss at 24 months

- Minimum lumen diameter

- Hospitalization

- Safety, defined as 30-day freedom from procedure-related death, unplanned amputation, and target lesion revascularization

- Restenosis pattern

- Target lesion revascularization, clinical success, and angiographic outcome

\begin{tabular}{ll}
\hline Starting date & 2012 \\
\hline Contact information & Dr. Gunnar Tepe (gunnar.tepe@ro-med.de) \\
\hline Notes & Sponsor: self-funded study \\
\hline
\end{tabular}

Drug-eluting balloon angioplasty versus uncoated balloon angioplasty for the treatment of in-stent restenosis of the femoropopliteal 


Trial name or title
in the femoral-popliteal artery: a randomized, controlled prospective study

\begin{tabular}{|c|c|}
\hline Methods & Study design: randomized controlled trial \\
\hline \multirow[t]{19}{*}{ Participants } & Country: China \\
\hline & Setting: Beijing PLA General Hospital \\
\hline & Number of participants: 120 \\
\hline & Inclusion criteria: \\
\hline & - Males and females $\geq 18$ years $<80$ years old \\
\hline & $\begin{array}{l}\text { - History of bare-metal stenting angioplasty in the femoral-popliteal artery for treatme } \\
\text { riosclerosis obliterans }\end{array}$ \\
\hline & - Intermittent claudication or critical limb ischemia 7 days after stenting angioplasty, 1 \\
\hline & Rutherford classification (category 2, 3, and 4) \\
\hline & $\begin{array}{l}\cdot \geq 50 \% \text { restenosis in the minimal luminal diameter within the stent-covered vessel, } \mathrm{pl} \\
\text { segments proximally and distally, vs the mean diameter stent measured by computed } \\
\text { angiography (CTA) }\end{array}$ \\
\hline & $\begin{array}{l}\text { - Single vessel runoff or better with at least } 1 \text { of } 3 \text { vessels (anterior tibial artery, posteri } \\
\text { artery, and peroneal artery) patent to the lower third of the calf }\end{array}$ \\
\hline & $\begin{array}{l}\text { - Treatment and follow-up should be voluntary and informed consent form would be } \\
\text { patient or a legal guardian }\end{array}$ \\
\hline & Exclusion criteria: \\
\hline & - Paclitaxel allergy or iodine allergy \\
\hline & $\cdot<18$ or $\geq 80$ years of age \\
\hline & - Current medical condition with life expectancy less than 3 months \\
\hline & - Symptomatic myocardial infarction within 3 months or uncontrollable heart failure \\
\hline & - Severe respiratory failure $\left(\mathrm{PO}_{2}<60 \mathrm{mmHg}\right.$ or $\left.\mathrm{PCO}_{2}>50 \mathrm{mmHg}\right)$ \\
\hline & - Renal failure with creatine level higher than $200 \mathrm{mmol} / \mathrm{L}$ \\
\hline & - Severe liver dysfunction with alanine aminotransferase level over 3 times \\
\hline
\end{tabular}

Vessels treated: femoropopliteal arteries

\section{Primary:}

- Luminal restenosis rate within the stent-covered vessel, plus 10-mm segments proximally and distally by Doppler ultrasound

- Peak systolic velocity ratio relative to adjacent segment

\section{Secondary:}

- Ankle brachial index

Drug-eluting balloon angioplasty versus uncoated balloon angioplasty for the treatment of in-stent restenosis of the femoropopliteal 
TRC-14004848 (Continued)

- Intimal thickness of lesion segment by Doppler ultrasound

- Pulse wave velocity

\begin{tabular}{ll}
\hline Starting date & Pending as of February 2017 \\
\hline Contact information & $\begin{array}{l}\text { Dr. Shen Chenyang (scy@pkuph.edu.cn), Department of Vascular Surgery, Peking University Peo- } \\
\text { ple's Hospital, } 11 \text { Xizhimen South Street, Xicheng District, Beijing, China, 100044 }\end{array}$ \\
\hline Notes & Sponsor: self-funded study \\
\hline
\end{tabular}

ALAT: alanine aminotransferase.

ASAT: aspartate aminotransferase.

CTA: computed tomography angiography.

DEB: drug-eluting balloon.

PAD: peripheral arterial disease.

$\mathrm{PCO}_{2}$ : partial pressure of carbon dioxide.

$\mathrm{PO}_{2}$ : partial pressure of oxygen.

SFA: superficial femoral artery.

\section{DATA AND ANALYSES}

\section{Comparison 1. Drug-eluting balloon (DEB) versus uncoated balloon angioplasty at 1 month}

\begin{tabular}{lllll}
\hline Outcome or subgroup title & No. of studies & $\begin{array}{l}\text { No. of partici- } \\
\text { pants }\end{array}$ & Statistical method & Effect size \\
\hline $\begin{array}{l}1 \text { Improvement } \geq 1 \text { Rutherford catego- } \\
\text { ry }\end{array}$ & 1 & 74 & $\begin{array}{l}\text { Odds Ratio (M-H, Fixed, 95\% } \\
\text { Cl) }\end{array}$ & 2.33 [0.55, 9.83] \\
\hline
\end{tabular}

Analysis 1.1. Comparison 1 Drug-eluting balloon (DEB) versus uncoated balloon
angioplasty at 1 month, Outcome 1 Improvement $\geq 1$ Rutherford category.

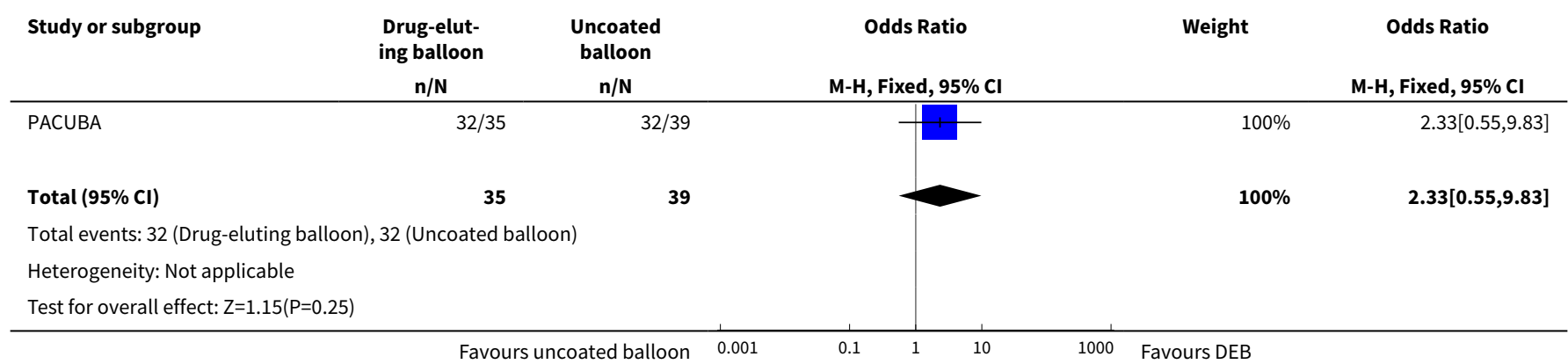


Comparison 2. Drug-eluting balloon (DEB) versus uncoated balloon angioplasty at 6 months

\begin{tabular}{llllll}
\hline Outcome or subgroup title & No. of studies & $\begin{array}{l}\text { No. of partici- } \\
\text { pants }\end{array}$ & Statistical method & Effect size \\
\hline $\begin{array}{l}1 \text { Target lesion revasculariza- } \\
\text { tion }\end{array}$ & 1 & 70 & Odds Ratio (M-H, Fixed, 95\% Cl) & $0.05[0.00,0.92]$ \\
\hline 2 Binary restenosis & 2 & 189 & Odds Ratio (M-H, Fixed, 95\% Cl) & $0.28[0.14,0.56]$ \\
\hline 3 Death & 3 & 263 & Odds Ratio (M-H, Fixed, 95\% Cl) & $0.93[0.13,6.71]$ \\
\hline $\begin{array}{l}4 \text { Improvement } \geq 1 \text { Ruther- } \\
\text { ford category }\end{array}$ & 2 & 193 & Odds Ratio (M-H, Fixed, 95\% Cl) & $1.81[1.02,3.21]$ \\
\hline
\end{tabular}

Analysis 2.1. Comparison 2 Drug-eluting balloon (DEB) versus uncoated balloon angioplasty at 6 months, Outcome 1 Target lesion revascularization.

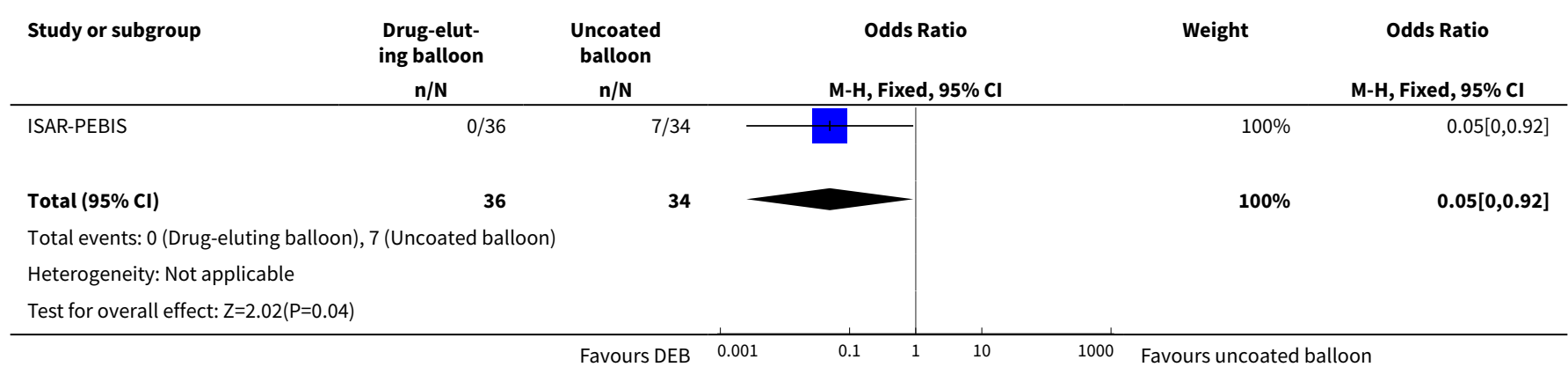

Analysis 2.2. Comparison 2 Drug-eluting balloon (DEB) versus uncoated balloon angioplasty at 6 months, Outcome 2 Binary restenosis.

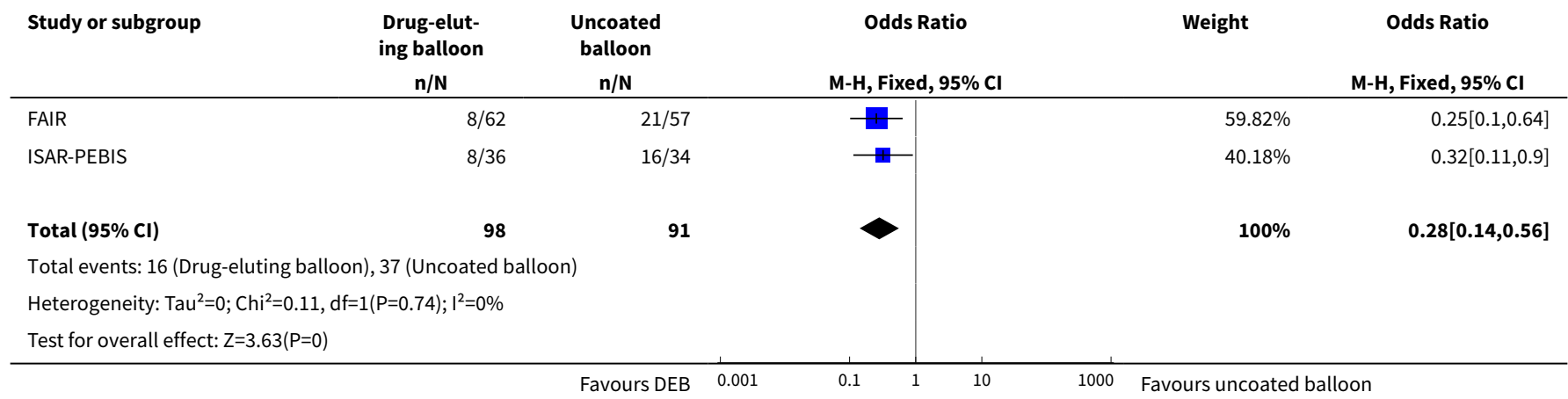


Analysis 2.3. Comparison 2 Drug-eluting balloon (DEB) versus uncoated balloon angioplasty at 6 months, Outcome 3 Death.

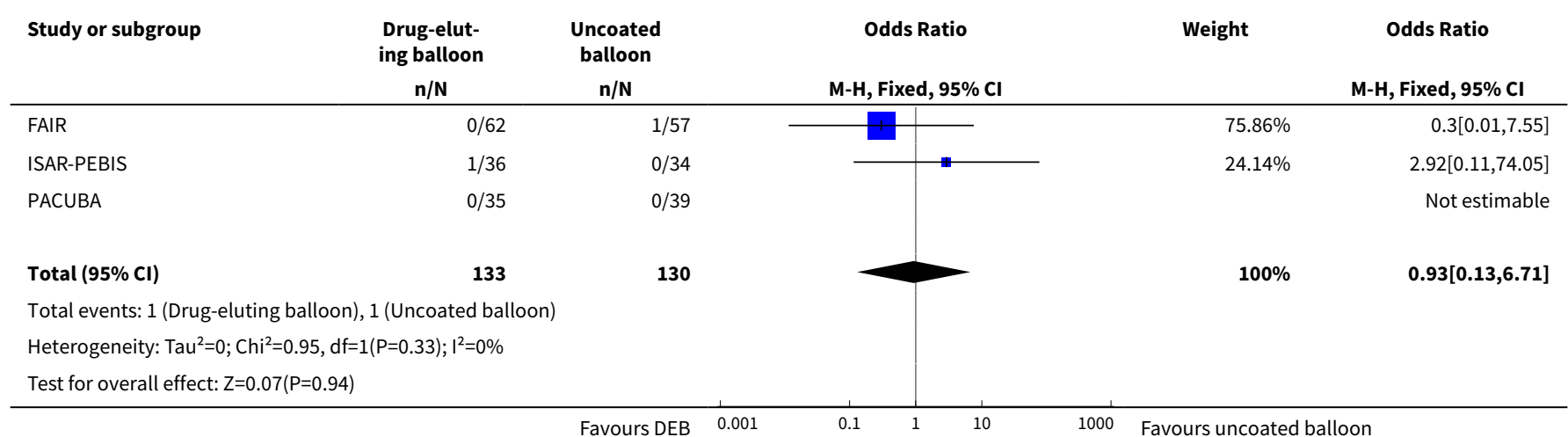

Analysis 2.4. Comparison 2 Drug-eluting balloon (DEB) versus uncoated balloon angioplasty at 6 months, Outcome 4 Improvement $\geq 1$ Rutherford category.

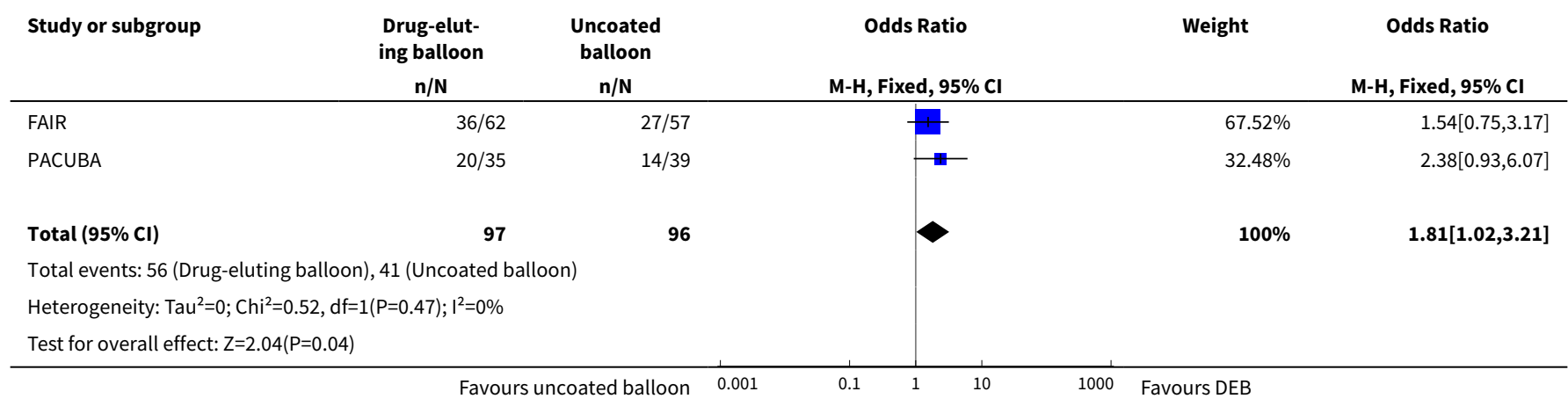

Comparison 3. Drug-eluting balloon (DEB) versus uncoated balloon angioplasty at 12 months

\begin{tabular}{lllll}
\hline Outcome or subgroup title & No. of studies & $\begin{array}{l}\text { No. of partici- } \\
\text { pants }\end{array}$ & Statistical method & Effect size \\
\hline 1 Binary restenosis & 1 & 119 & Odds Ratio (M-H, Fixed, 95\% Cl) & $0.34[0.15,0.76]$ \\
\hline 2 Death & 2 & 193 & Odds Ratio (M-H, Fixed, 95\% Cl) & $0.6[0.10,3.73]$ \\
\hline $\begin{array}{l}3 \text { Improvement } \geq 1 \text { Ruther- } \\
\text { ford category }\end{array}$ & 2 & 193 & Odds Ratio (M-H, Fixed, 95\% Cl) & $2.08[1.13,3.83]$ \\
\hline
\end{tabular}


Analysis 3.1. Comparison 3 Drug-eluting balloon (DEB) versus uncoated balloon angioplasty at 12 months, Outcome 1 Binary restenosis.

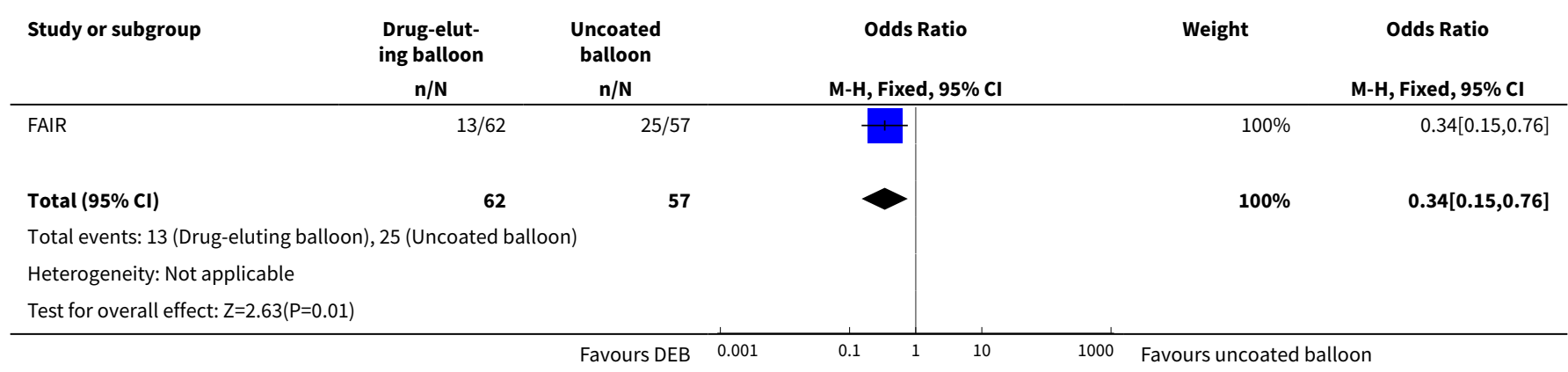

Analysis 3.2. Comparison 3 Drug-eluting balloon (DEB) versus uncoated balloon angioplasty at 12 months, Outcome 2 Death.

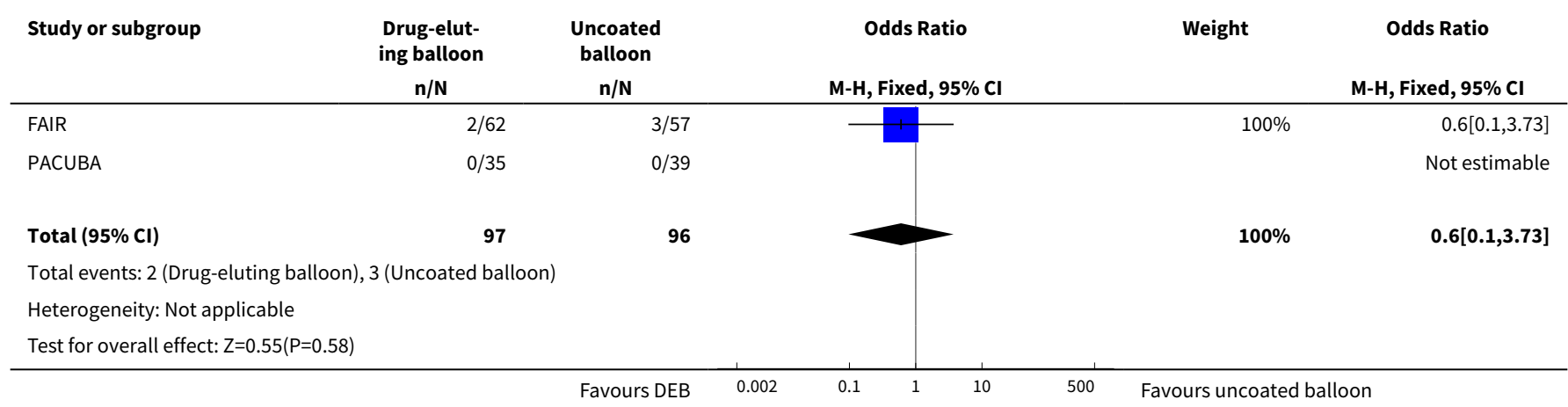

Analysis 3.3. Comparison 3 Drug-eluting balloon (DEB) versus uncoated balloon angioplasty at 12 months, Outcome 3 Improvement $\geq 1$ Rutherford category.

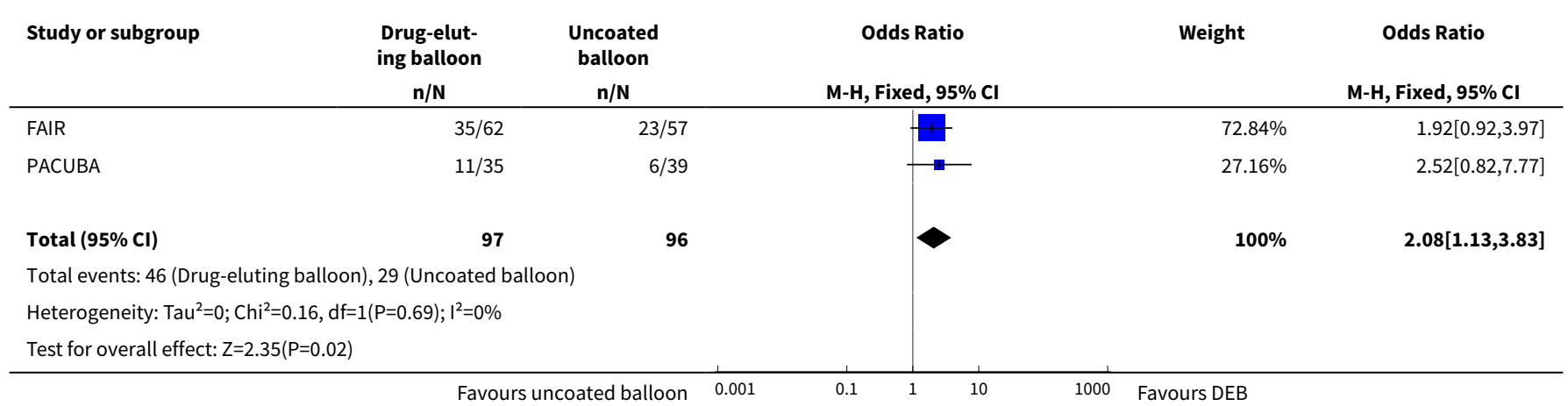


Comparison 4. Drug-eluting balloon (DEB) versus uncoated balloon angioplasty at 24 months

\begin{tabular}{lllll}
\hline Outcome or subgroup title & No. of studies & $\begin{array}{l}\text { No. of partici- } \\
\text { pants }\end{array}$ & Statistical method & Effect size \\
\hline $\begin{array}{l}1 \text { Target lesion revascular- } \\
\text { ization }\end{array}$ & 1 & 70 & Odds Ratio (M-H, Fixed, 95\% Cl) & $0.24[0.08,0.70]$ \\
\hline 2 Death & 1 & 70 & Odds Ratio (M-H, Fixed, 95\% Cl) & $7.21[0.36,144.95]$ \\
\hline
\end{tabular}

Analysis 4.1. Comparison 4 Drug-eluting balloon (DEB) versus uncoated balloon angioplasty at 24 months, Outcome 1 Target lesion revascularization.

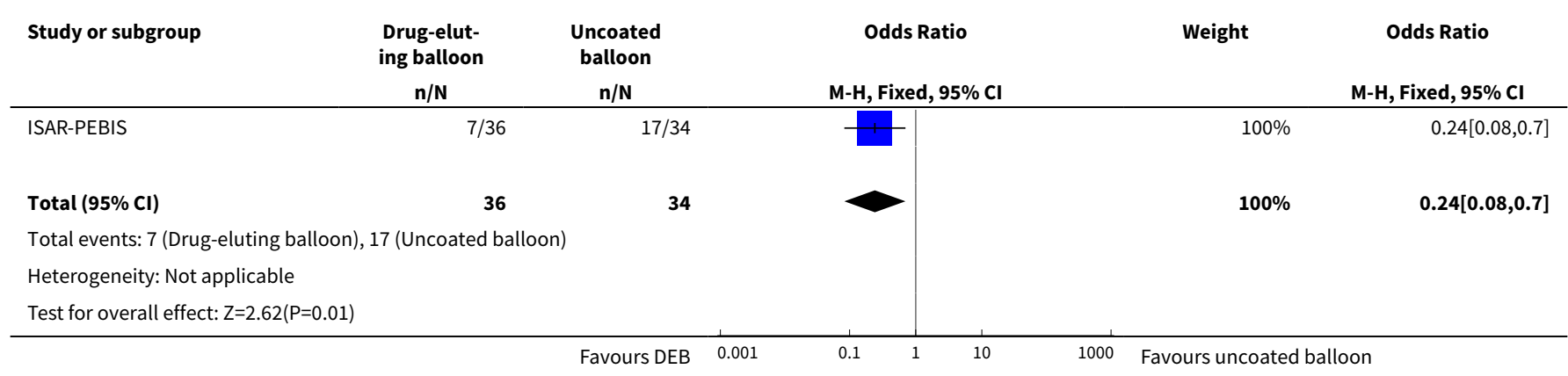

Analysis 4.2. Comparison 4 Drug-eluting balloon (DEB) versus uncoated balloon angioplasty at 24 months, Outcome 2 Death.

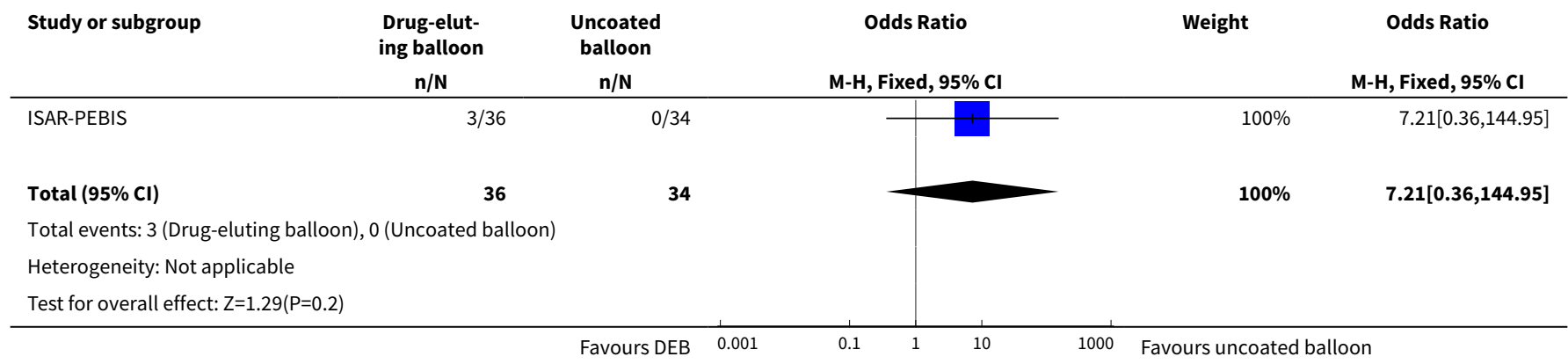

\section{A P PEN DICES}

Appendix 1. CENTRAL search strategy, February 2, 2017

Search run on Thu Feb 22017

\begin{tabular}{lll}
\hline$\# 1$ & MESH DESCRIPTOR Arteriosclerosis & 868 \\
\hline
\end{tabular}

Drug-eluting balloon angioplasty versus uncoated balloon angioplasty for the treatment of in-stent restenosis of the femoropopliteal 
(Continued)

\begin{tabular}{|c|c|c|}
\hline \#2 & MESH DESCRIPTOR Arteriosclerosis Obliterans & 71 \\
\hline \#3 & MESH DESCRIPTOR Atherosclerosis & 619 \\
\hline \#4 & MESH DESCRIPTOR Arterial Occlusive Diseases & 724 \\
\hline \#5 & MESH DESCRIPTOR Intermittent Claudication & 712 \\
\hline \#6 & MESH DESCRIPTOR Ischemia & 789 \\
\hline \#7 & MESH DESCRIPTOR Peripheral Vascular Diseases EXPLODE ALL TREES & 2201 \\
\hline \#8 & MESH DESCRIPTOR Leg EXPLODE ALL TREES WITH QUALIFIERS BS & 1107 \\
\hline \#9 & MESH DESCRIPTOR Iliac Artery & 144 \\
\hline \#10 & MESH DESCRIPTOR Popliteal Artery & 278 \\
\hline \#11 & MESH DESCRIPTOR Femoral Artery & 810 \\
\hline \#12 & MESH DESCRIPTOR Tibial Arteries & 33 \\
\hline \#13 & (atherosclero* or arteriosclero* or PVD or PAOD or PAD ):TI,AB,KY & 9119 \\
\hline \#14 & $\begin{array}{l}\left.\text { ((arter }{ }^{\star} \text { or vascular or vein } \text { or veno }^{\star} \text { or peripher }{ }^{\star}\right) \text { near3 }\left(\text { occlus }^{\star} \text { or reocclus }\right. \\
\text { or re-occlus } \\
\left.\text { or stiffen } \text { or }^{\star} \text { or obliteno }{ }^{\star}\right) \text { ) } \text { or } \mathrm{TI}, \mathrm{AB}, \mathrm{KY}\end{array}$ & 7966 \\
\hline \#15 & (peripheral near3 dis*):TI,AB,KY & 3371 \\
\hline \#16 & (claudic* or IC):TI,AB,KY & 3063 \\
\hline$\# 17$ & (isch* or $\mathrm{CLI}$ ):TI,AB,KY & 23713 \\
\hline$\# 18$ & arteriopathic:TI,AB,KY & 7 \\
\hline$\# 19$ & dysvascular*:TI,AB,KY & 10 \\
\hline \#20 & leg:TI,AB,KY & 11505 \\
\hline$\# 21$ & (lower limb):TI,AB,KY & 2710 \\
\hline \#22 & (lower extrem $\left.{ }^{\star}\right): \mathrm{TI}, \mathrm{AB}, \mathrm{KY}$ & 3406 \\
\hline \#23 & (above knee):TI,AB,KY & 125 \\
\hline \#24 & (below knee):TI,AB,KY & 248 \\
\hline \#25 & $\begin{array}{l}\text { (femor* or iliac or popliteal or fempop* or crural or poplite* or infrapopliteal or } \\
\text { inguinal or femdist* or infrainquinal or tibial):TI,AB,KY }\end{array}$ & 13629 \\
\hline \#26 & restenosis:TI,AB,KY & 2489 \\
\hline$\# 27$ & $\begin{array}{l}\# 1 \text { OR \#2 OR \#3 OR \#4 OR \#5 OR \#6 OR \#7 OR \#8 OR \#9 OR \#10 OR \#11 OR \#12 } \\
\text { OR \#13 OR \#14 OR \#15 OR \#16 OR \#17 OR \#18 OR \#19 OR \#20 OR \#21 OR \#22 OR } \\
\text { \#23 OR \#24 OR \#25 OR \#26 }\end{array}$ & 66811 \\
\hline
\end{tabular}

Drug-eluting balloon angioplasty versus uncoated balloon angioplasty for the treatment of in-stent restenosis of the femoropopliteal 
(Continued)

\begin{tabular}{|c|c|c|}
\hline \#28 & MESH DESCRIPTOR Angioplasty EXPLODE ALL TREES & 4144 \\
\hline$\# 29$ & MESH DESCRIPTOR Endovascular Procedures & 203 \\
\hline$\# 30$ & (angioplas ${ }^{\star}$ or percutan ${ }^{\star}$ or $\mathrm{PTA}$ ):TI,AB,KY & 13449 \\
\hline \#31 & valvuloplasty:TI,AB,KY & 133 \\
\hline \#32 & (recanali* or revascular $\left.{ }^{\star}\right): \mathrm{TI}, \mathrm{AB}, \mathrm{KY}$ & 7386 \\
\hline \#33 & dilat $^{\star}: T I, A B, K Y$ & 7493 \\
\hline \#34 & (balloon or baloon):TI,AB,KY & 6925 \\
\hline \#35 & endovascular:TI,AB,KY & 1510 \\
\hline \#36 & \#28 OR \#29 OR \#30 OR \#31 OR \#32 OR \#33 OR \#34 OR \#35 & 26979 \\
\hline \#37 & MESH DESCRIPTOR Paclitaxel & 1519 \\
\hline \#38 & (coated or uncoated):TI,AB,KY & 3677 \\
\hline \#39 & (drug and (elut* or releas*)):TI,AB,KY & 20097 \\
\hline$\# 40$ & DEB:TI,AB,KY & 97 \\
\hline \#41 & POBA:TI,AB,KY & 44 \\
\hline \#42 & paclitax*:TI,AB,KY & 4384 \\
\hline \#43 & sirolimus:TI,AB,KY & 2036 \\
\hline \#44 & zotarolimus:TI,AB,KY & 316 \\
\hline \#45 & rapalog:TI,AB,KY & 1 \\
\hline \#46 & $\begin{array}{l}\text { (IN.PACT or MOXY or Paseo or Lutonix or Paccoca or PANTERA or ELUTAX or } \\
\text { DIOR or FREEWAY or SeQuent or GENIE):TI,AB,KY }\end{array}$ & 85 \\
\hline \#47 & \#37 OR \#38 OR \#39 OR \#40 OR \#41 OR \#42 OR \#43 OR \#44 OR \#45 OR \#46 & 28056 \\
\hline \#48 & \#27 AND \#36 AND \#47 & 1524 \\
\hline \#49 & ${ }^{\star}$ NOT SR-PVD:CC & 979221 \\
\hline \#50 & \#48 AND \#49 & 1417 \\
\hline
\end{tabular}

\section{Appendix 2. Trial registries searches, February 2, 2017}

ClinicalTrials.gov

95 studies found for: restenosis AND peripheral arterial AND randomized

World Health Organization International Clinical Trials Registry Platform

57 records for 56 trials found for: angioplasty and restenosis

Drug-eluting balloon angioplasty versus uncoated balloon angioplasty for the treatment of in-stent restenosis of the femoropopliteal 
ISRCTN Register

24 results angioplasty and restenosis

Appendix 3. Database searches, November 28, 2017

\begin{tabular}{|c|c|c|}
\hline Source & Search strategy & Hits retrieved \\
\hline \multirow[t]{26}{*}{ CENTRAL } & \#1 MESH DESCRIPTOR Arteriosclerosis 872 & 336 \\
\hline & \#2 MESH DESCRIPTOR Arteriosclerosis Obliterans 73 & \\
\hline & \#3 MESH DESCRIPTOR Atherosclerosis 684 & \\
\hline & \#4 MESH DESCRIPTOR Arterial Occlusive Diseases 746 & \\
\hline & \#5 MESH DESCRIPTOR Intermittent Claudication 738 & \\
\hline & \#6 MESH DESCRIPTOR Ischemia 823 & \\
\hline & \#7 MESH DESCRIPTOR Peripheral Vascular Diseases EXPLODE ALL TREES 2288 & \\
\hline & \#8 MESH DESCRIPTOR Iliac Artery 154 & \\
\hline & \#9 MESH DESCRIPTOR Femoral Artery 873 & \\
\hline & \#10 MESH DESCRIPTOR Tibial Arteries 35 & \\
\hline & \#11 (atherosclero* or arteriosclero* or PVD or PAOD or PAD ):TI,AB,KY 10322 & \\
\hline & 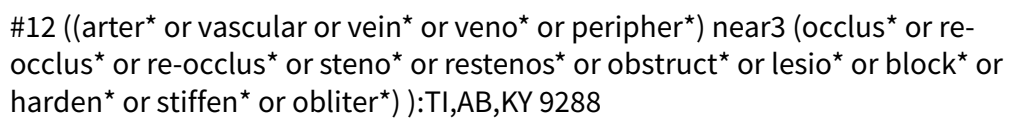 & \\
\hline & \#13 (peripheral near3 dis*):TI,AB,KY 3862 & \\
\hline & \#14 (claudic* or IC):TI,AB,KY 3571 & \\
\hline & \#15 (isch* or CLI):TI,AB,KY 27272 & \\
\hline & $\# 16$ arteriopathic: $\mathrm{TI}, \mathrm{AB}, \mathrm{KY} 7$ & \\
\hline & \#17 dysvascular*:TI,AB,KY 12 & \\
\hline & \#18 leg:TI,AB,KY 12842 & \\
\hline & \#19 (lower limb):TI,AB,KY 3335 & \\
\hline & \#20 (lower extrem*):TI,AB,KY 3915 & \\
\hline & \#21 (above knee):TI,AB,KY 133 & \\
\hline & \#22 (below knee):TI,AB,KY 263 & \\
\hline & $\begin{array}{l}\text { \#23 (femor* or iliac or popliteal or fempop* or crural or poplite* or in- } \\
\text { frapopliteal or inguinal or femdist* or infrainquinal or tibial):TI,AB,KY } 15799\end{array}$ & \\
\hline & \#24 restenosis:TI,AB,KY 2771 & \\
\hline & \#25 MESH DESCRIPTOR Popliteal Artery 294 & \\
\hline & \#26 MESH DESCRIPTOR leg 2742 & \\
\hline
\end{tabular}

Drug-eluting balloon angioplasty versus uncoated balloon angioplasty for the treatment of in-stent restenosis of the femoropopliteal 
\#27 \#1 OR \#2 OR \#3 OR \#4 OR \#5 OR \#6 OR \#7 OR \#8 OR \#9 OR \#10 OR \#11 OR \#12 OR \#13 OR \#14 OR \#15 OR \#16 OR \#17 OR \#18 OR \#19 OR \#20 OR \#21 OR \#22 OR \#23 OR \#24 OR \#25 OR \#26 76401

\section{\#28 MESH DESCRIPTOR Angioplasty EXPLODE ALL TREES 4239}

\#29 MESH DESCRIPTOR Endovascular Procedures 261

\#30 (angioplas* or percutan* or PTA):TI,AB,KY 15653

\#31 valvuloplasty:TI,AB,KY 168

\#32 ((recanali* or revascular*)):TI,AB,KY 8613

\#33 dilat*:TI,AB,KY 8348

\#34 (balloon or baloon):TI,AB,KY 7629

\#35 endovascular:TI,AB,KY 2099

\#36 \#28 OR \#29 OR \#30 OR \#31 OR \#32 OR \#33 OR \#34 OR \#35 31256

\#37 MESH DESCRIPTOR Paclitaxel 1667

\#38 (coated or uncoated):TI,AB,KY 4046

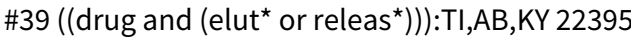

\#40 DEB:TI,AB,KY 141

\#41 POBA:TI,AB,KY 60

\#42 paclitax*:TI,AB,KY 5425

\#43 sirolimus:TI,AB,KY 2291

\#44 zotarolimus:TI,AB,KY 382

\#45 rapalog:TI,AB,KY 2

\#46 ((IN.PACT or MOXY or Paseo or Lutonix or Paccoca or PANTERA or ELUTAX or DIOR or FREEWAY or SeQuent or GENIE)):TI,AB,KY 101

\#47 \#37 OR \#38 OR \#39 OR \#40 OR \#41 OR \#42 OR \#43 OR \#44 OR \#45 OR \#46 31639

\#48 \#27 AND \#36 AND \#47 1864

\#49 * NOT SR-PVD:CC 1088498

\#50 \#48 AND \#49 1753

\#51 01/01/2017 TO 28/11/2017:CD 109501

\#52 \#50 AND \#51 336

Clinicaltrials.gov $\quad$ randomized | peripheral arterial | restenosis | First posted from 01/01/2017 to 1
$11 / 28 / 2017$ | Last update posted from $01 / 01 / 2017$ to $11 / 28 / 2017$

\begin{tabular}{lll}
\hline ICTRP Search Portal & angioplasty and restenosis 01/01/2017 to 28/11/2017 & 1 \\
\hline MEDLINE & 1 Arteriosclerosis/ 60027 & 238 \\
& 2 Arteriosclerosis Obliterans/4192 \\
& 3 Atherosclerosis/ 32486 \\
\hline
\end{tabular}

Drug-eluting balloon angioplasty versus uncoated balloon angioplasty for the treatment of in-stent restenosis of the femoropopliteal 36 arteries (Review)

Copyright $\odot 2019$ The Cochrane Collaboration. Published by John Wiley \& Sons, Ltd. 


\author{
4 Arterial Occlusive Diseases/ 28247 \\ 5 Intermittent Claudication/ 8184 \\ 6 Ischemia/ 50319 \\ 7 exp Peripheral Vascular Diseases/ 53026 \\ 8 exp Leg/bs [Blood Supply] 26583 \\ 9 Iliac Artery/ 14224
}

10 Popliteal Artery/ 9465

11 Femoral Artery/ 28635

12 Tibial Arteries/ 1574

13 ((arter ${ }^{\star}$ or vascular or vein ${ }^{\star}$ or veno* or peripher*) adj3 (occlus* or reocclus* or re-occlus ${ }^{\star}$ or steno* or restenos ${ }^{\star}$ or obstruct $^{\star}$ or lesio* or block ${ }^{\star}$ or harden* or stiffen* or obliter $\left.{ }^{\star}\right)$ ).ti,ab. 151212

14 (peripheral adj3 dis ${ }^{\star}$ ).ti,ab. 39838

15 (atherosclero* or arteriosclero* or PVD or PAOD or PAD).ti,ab. 179677

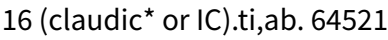

17 (isch* or CLI).ti,ab. 364590

18 arteriopathic.ti,ab. 181

19 dysvascular ${ }^{\star}$. ti,ab. 222

20 leg.ti,ab. 85167

21 lower limb.ti,ab. 29009

22 lower extrem ${ }^{\star} . \mathrm{ti}, \mathrm{ab} .49907$

23 above knee.ti,ab. 1924

24 below knee.ti,ab. 2912

25 (femor* or iliac or popliteal or fempop* or crural or poplite* or infrapopliteal or inguinal or femdist ${ }^{\star}$ or infrainquinal or tibial).ti,ab. 243299

26 restenosis.ti,ab. 21527

27 or/1-26 1146562

28 exp Angioplasty/ 62714

29 Endovascular Procedures/ 13305

30 (angioplas* or percutan* or PTA).ti,ab. 166701

31 valvuloplasty.ti,ab. 4554

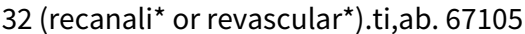

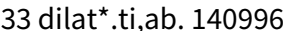

34 (balloon or baloon).ti,ab. 61847

35 endovascular.ti,ab. 42459

36 or/28-35 426689

Drug-eluting balloon angioplasty versus uncoated balloon angioplasty for the treatment of in-stent restenosis of the femoropopliteal arteries (Review)

Copyright $\odot 2019$ The Cochrane Collaboration. Published by John Wiley \& Sons, Ltd. 


\section{Paclitaxel/ 25241}

38 (coated or uncoated).ti,ab. 100498

39 (drug and (elut* or releas $\left.{ }^{\star}\right)$ ).ti,ab. 89291

40 DEB.ti,ab. 1321

41 POBA.ti,ab. 202

42 paclitax*.ti,ab. 26659

43 sirolimus.ti,ab. 7247

44 zotarolimus.ti,ab. 619

45 rapalog.ti,ab. 84

46 (INPACT or MOXY or Paseo or Lutonix or Paccoca or PANTERA or ELUTAX or DIOR or FREEWAY or SeQuent or GENIE).ti,ab. 1092

47 or/37-46 223743

4827 and 36 and 475834

49 randomized controlled trial.pt. 505458

50 controlled clinical trial.pt. 100426

51 randomized.ab. 442421

52 placebo.ab. 205527

53 drug therapy.fs. 2147127

54 randomly.ab. 305346

55 trial.ab. 466105

56 groups.ab. 1885958

57 or/49-56 4449754

58 exp animals/ not humans.sh. 4743200

5957 not 583848554

6048 and 592607

$612017^{\star}$.ed. 953719

6260 and 61238

2 Arteriosclerosis Obliterans/ 11174

3 Atherosclerosis/ 111897

4 Arterial Occlusive Diseases/ 5724

5 Intermittent Claudication/ 5974

6 Ischemia/ 58464

7 exp Peripheral Vascular Diseases/ 1250931

Drug-eluting balloon angioplasty versus uncoated balloon angioplasty for the treatment of in-stent restenosis of the femoropopliteal 


\author{
8 Iliac Artery/ 9653 \\ 9 Popliteal Artery/ 5112 \\ 10 Femoral Artery/ 20342 \\ 11 Tibial Arteries/ 2034 \\ 12 ((arter ${ }^{\star}$ or vascular or vein ${ }^{\star}$ or veno* or peripher $^{\star}$ ) adj3 (occlus* or reocclus ${ }^{\star}$ \\ or re-occlus ${ }^{\star}$ or steno* or restenos ${ }^{\star}$ or obstruct ${ }^{\star}$ or lesio* or block ${ }^{\star}$ or harden ${ }^{\star}$ \\ or stiffen ${ }^{\star}$ or obliter $\left.{ }^{\star}\right)$ ).ti,ab. 140600 \\ 13 (peripheral adj3 dis ${ }^{\star}$ ).ti,ab. 42066 \\ 14 (atherosclero* or arteriosclero* or PVD or PAOD or PAD).ti,ab. 188142

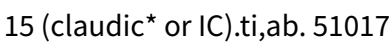 \\ 16 (isch* or CLI).ti,ab. 388806 \\ 17 arteriopathic.ti,ab. 82 \\ 18 dysvascular*.ti,ab. 168 \\ 19 leg.ti,ab. 78344
}

20 lower limb.ti,ab. 32148

21 lower extrem*.ti,ab. 48968

22 above knee.ti,ab. 1407

23 below knee.ti,ab. 2163

24 (femor ${ }^{\star}$ or iliac or popliteal or fempop* or crural or poplite* or infrapopliteal or inguinal or femdist* or infrainquinal or tibial).ti,ab. 223048

25 restenosis.ti,ab. 26376

26 exp Angioplasty/ 67815

27 Endovascular Procedures/ 19628

28 (angioplas* or percutan* or PTA).ti,ab. 186707

29 valvuloplasty.ti,ab. 4263

30 (recanali $^{\star}$ or revascular ${ }^{\star}$ ).ti,ab. 80742

31 dilat* $^{*}$ ti,ab. 143183

32 (balloon or baloon).ti,ab. 69520

33 endovascular.ti,ab. 56266

34 or/26-33 471247

35 Paclitaxel/ 87997

36 (coated or uncoated).ti,ab. 87359

37 (drug and (elut* or releas*)).ti,ab. 107786

38 DEB.ti,ab. 2103

39 POBA.ti,ab. 502

40 paclitax ${ }^{\star}$.ti,ab. 38355

Drug-eluting balloon angioplasty versus uncoated balloon angioplasty for the treatment of in-stent restenosis of the femoropopliteal

Copyright $\odot 2019$ The Cochrane Collaboration. Published by John Wiley \& Sons, Ltd. 
41 sirolimus.ti,ab. 12272

42 zotarolimus.ti,ab. 1541

43 rapalog.ti,ab. 132

44 (INPACT or MOXY or Paseo or Lutonix or Paccoca or PANTERA or ELUTAX or DIOR or FREEWAY or SeQuent or GENIE).ti,ab. 1671

45 or/35-44 283715

46 or/1-25 1781664

4734 and 45 and 4616133

48 randomized controlled trial/ 436425

49 controlled clinical trial/ 408134

50 random\$.ti,ab. 1129645

51 randomization/ 68144

52 intermethod comparison/ 223370

53 placebo.ti,ab. 214681

54 (compare or compared or comparison).ti. 326079

55 ((evaluated or evaluate or evaluating or assessed or assess) and (compare or compared or comparing or comparison)).ab. 1558492

56 (open adj label).ti,ab. 59910

57 ((double or single or doubly or singly) adj (blind or blinded or blindly)).ti,ab. 153101

58 double blind procedure/ 119054

59 parallel group\$1.ti,ab. 18934

60 (crossover or cross over).ti,ab. 70012

61 ((assign\$ or match or matched or allocation) adj5 (alternate or group\$1 or intervention $\$ 1$ or patient $\$ 1$ or subject $\$ 1$ or participant $\$ 1)$ ).ti,ab. 240625

62 (assigned or allocated).ti,ab. 281778

63 (controlled adj7 (study or design or trial)).ti,ab. 252369

64 (volunteer or volunteers).ti,ab. 167849

65 trial.ti. 205628

66 or/48-65 3366239

6747 and 667694

$682017^{\star}$. dc. 1656576

6967 and 68633

\begin{tabular}{ll}
\hline AMED & 1 Arteriosclerosis/ 78 \\
& 2 Atherosclerosis/ 209 \\
& 3 Intermittent Claudication/ 72
\end{tabular}

Drug-eluting balloon angioplasty versus uncoated balloon angioplasty for the treatment of in-stent restenosis of the femoropopliteal 40 arteries (Review)

Copyright @ 2019 The Cochrane Collaboration. Published by John Wiley \& Sons, Ltd. 


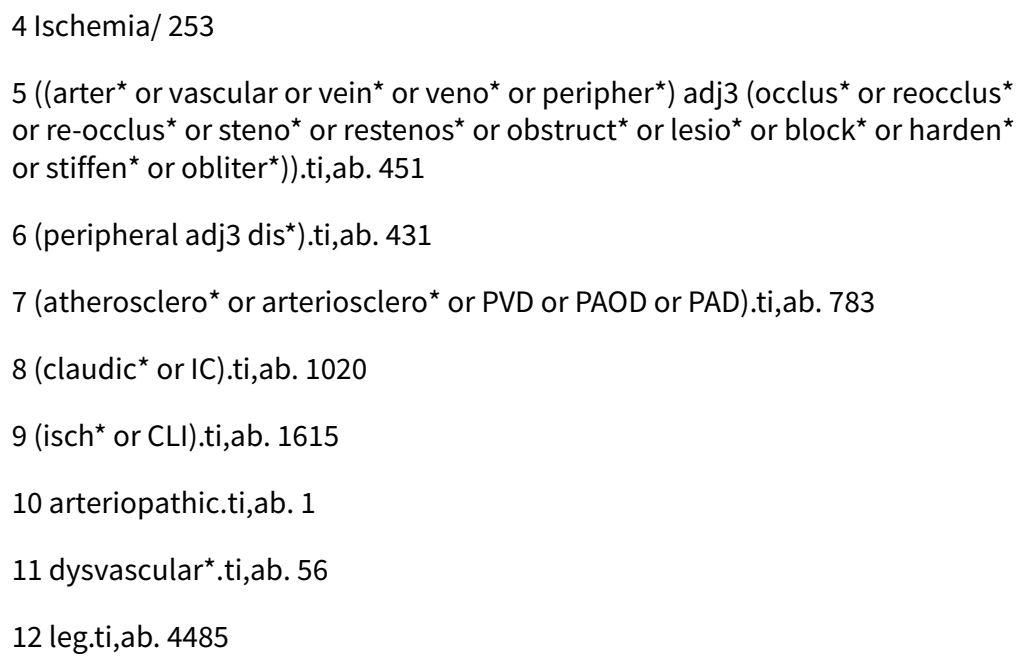

10 arteriopathic.ti,ab. 1

11 dysvascular*.ti,ab. 56

12 leg.ti,ab. 4485

13 lower limb.ti,ab. 2285

14 lower extrem*.ti,ab. 3083

15 above knee.ti,ab. 142

16 below knee.ti,ab. 353

17 (femor ${ }^{\star}$ or iliac or popliteal or fempop* or crural or poplite* or infrapopliteal or inguinal or femdist* ${ }^{\star}$ or infrainquinal or tibial).ti,ab. 4656

18 restenosis.ti,ab. 35

19 exp Angioplasty/ 16

20 (angioplas* or percutan* or PTA).ti,ab. 765

21 valvuloplasty.ti,ab. 6

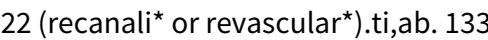

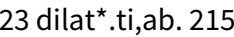

24 (balloon or baloon).ti,ab. 89

25 endovascular.ti,ab. 28

26 (coated or uncoated).ti,ab. 117

27 (drug and (elut ${ }^{\star}$ or releas $\left.{ }^{\star}\right)$ ).ti,ab. 115

28 DEB.ti,ab. 1

29 paclitax ${ }^{\star} . t i, a b .79$

30 (INPACT or MOXY or Paseo or Lutonix or Paccoca or PANTERA or ELUTAX or DIOR or FREEWAY or SeQuent or GENIE).ti,ab. 17

31 or/1-18 16723

32 or/19-25 1169

33 or/26-30 328

3431 and 32 and 332

Drug-eluting balloon angioplasty versus uncoated balloon angioplasty for the treatment of in-stent restenosis of the femoropopliteal 
S53 S47 OR S48 OR S49 OR S50 OR S51 OR S52 951,324

S52 TX randomly 41,642

S51 TX "treatment as usual" 709

S50 TX "double-blind" 755,121

S49 TX "single-blind*" 8,665

S48 TX trial 236,355

S47 MH "Clinical Trials" 90,798

S46 S26 AND S34 AND S45 1,583

S45 S35 OR S36 OR S37 OR S38 OR S39 OR S40 OR S41 OR S42 OR S43 OR S44 21,062

S44 TX IN.PACT or MOXY or Paseo or Lutonix or Paccoca or PANTERA or ELUTAX or DIOR or FREEWAY or SeQuent or GENIE 513

\section{S43 TX rapalog 6}

S42 TX zotarolimus 127

S41 TX sirolimus 2,040

S40 TX paclitax 3,181

S39 TX POBA 13

S38 TX DEB 1,259

S37 TX (drug and (elut* or releas $\left.{ }^{\star}\right)$ ) 13,648

S36 TX coated or uncoated 2,367

S35 (MH "Paclitaxel") 2,289

S34 S27 OR S28 OR S29 OR S30 OR S31 OR S32 OR S33 42,521

S33 TX endovascular 4,527

S32 TX balloon or baloon 6,457

S31 TX dilat* 9,790

S30 TX (recanali* or revascular $\left.{ }^{\star}\right) 7,744$

S29 TX valvuloplasty 297

S28 TX angioplas * or percutan* or PTA 22,778

S27 (MH "Angioplasty+") 8,833

S26 S1 OR S2 OR S3 OR S4 OR S5 OR S6 OR S7 OR S8 OR S9 OR S10 OR S11 OR S12 OR S13 OR S14 OR S15 OR S16 OR S17 OR S18 OR S19 OR S20 OR S21 OR S22 OR S23 OR S24 OR S25 135,601

S25 TX restenosis 2,254

S24 TX femor* or iliac or popliteal or fempop* or crural or poplite* or infrapopliteal or inguinal or femdist* or infrainquinal or tibial 24,317 
S22 TX above knee 754

S21 TX lower extrem ${ }^{\star} 13,945$

S20 TX lower limb 7,329

S19 TX leg 23,610

S18 TX dysvascular* 165

S17 TX arteriopathic 10

S16 TX isch* or CLI 37,878

S15 TX claudic* or IC 6,833

S14 TX (peripheral n3 dis*) 8,837

S13 TX ((arter* or vascular or vein* or veno* or peripher ${ }^{\star}$ ) n3 (occlus ${ }^{\star}$ or reocclus $^{\star}$ or re-occlus ${ }^{\star}$ or steno* or restenos ${ }^{\star}$ or obstruct ${ }^{\star}$ or lesio* or block* or harden* or stiffen ${ }^{\star}$ or obliter $\left.{ }^{\star}\right)$ 12,203

S12 TX atherosclero* or arteriosclero* or PVD or PAOD or PAD 25,428

S11 (MH "Tibial Arteries") 134

S10 (MH "Femoral Artery") 1,180

S9 (MH "Popliteal Artery") 352

S8 (MH "Iliac Artery") 449

S7 (MH "Leg/BS") 441

S6 (MH "Peripheral Vascular Diseases") 2,952

S5 (MH "Ischemia") 3,239

S4 (MH "Intermittent Claudication") 831

S3 (MH "Arterial Occlusive Diseases") 1,581

S2 (MH "Atherosclerosis") 3,139

S1 (MH "Arteriosclerosis") 4,830

\section{CONTRIBUTIONS OF AUTHORS}

AK: protocol drafting, acquisition of trial reports, trial selection, data extraction, data analysis, data interpretation, review drafting, future review updates, guarantor of the review.

WAJ: protocol drafting, acquisition of trial reports, trial selection, data extraction, data analysis, data interpretation, review drafting. GP: data interpretation, review drafting. DK: data interpretation, review drafting. TF: data interpretation, review drafting. DR: data interpretation, review drafting. RN: data interpretation, review drafting. $A D$ : data interpretation, review drafting, future review updates.

\section{DECLARATIONS OF INTEREST}

AK: none known.

WAJ: none known.

Drug-eluting balloon angioplasty versus uncoated balloon angioplasty for the treatment of in-stent restenosis of the femoropopliteal

Copyright $\odot 2019$ The Cochrane Collaboration. Published by John Wiley \& Sons, Ltd. 
GP: received consultancy fees from WL Gore for acting as a reviewer of complications for a study involving an endovascular device and for support for speaking/lectures. He has received a grant from Gore for creation of a database for endovascular aneurysm repair research and from Abbott for development of a database for peripheral endovascular procedures. He has received honoraria from WL Gore and Medtronic for lectures and for speaking.

DK: none known.

TF: none known. Reports that his host institution, the University of Toronto Division of Vascular Surgery, receives academic support from several industry partners (Cook Medical, Medtronic, Liva Nova, CHS, Lemaitre, Gore) in the form of unrestricted educational grants.

DR: received consultancy fees for lectures around dialysis interventions and support for a live case course from CR Bard.

RN: has declared that he received payment for roles within the Scientific Advisory Boards of Graftworx and WL Gore; and from Fresenius for presenting an educational program for podiatric audiences and for legal defence malpractice work. He has no financial relationships related to the topic of this review.

AD: none known.

\section{SOURCES OF SUPPORT}

\section{Internal sources}

- No sources of support supplied

\section{External sources}

- Chief Scientist Office, Scottish Government Health Directorates, The Scottish Government, UK.

The Cochrane Vascular editorial base is supported by the Chief Scientist Office.

\section{DIFFERENCES BETWEEN PROTOCOLAND REVIEW}

The protocol identified numerous anatomical and clinical outcomes that were not reported by any of the included trials. Because it was reported in two of the included studies, the review included outcomes such as $A B I$ values, rather than change in $A B I$, and freedom from TLR, which we had not identified as an outcome of interest in the protocol. Unfortunately, it is not possible to perform any subgroup or sensitivity analyses as had been intended in the protocol because of the small number of included studies.

\section{N O T E S}

Parts of the methods section of this review are based on a standard template established by Cochrane Vascular.

\section{N DEX TERMS}

\section{Medical Subject Headings (MeSH)}

${ }^{\star}$ Femoral Artery; *Popliteal Artery; *Stents; Amputation; Angioplasty, Balloon [adverse effects] [ ${ }^{\star}$ methods]; Ankle Brachial Index; Constriction, Pathologic [etiology] [mortality] [therapy]; Randomized Controlled Trials as Topic; Recurrence; Vascular Patency

\section{MeSH check words}

Humans 